\title{
Nonparametric Mean Curvature Flow with Nearly Vertical Contact Angle Condition
}

\author{
Zhenghuan $\mathrm{Gao}^{1, *}$, Xinan Ma1 ${ }^{1}$ Peihe Wang ${ }^{2}$ and Liangjun Weng ${ }^{1,3}$ \\ 1 School of Mathematical Sciences, University of Science and Technology of China, \\ Hefei 230026, China; \\ 2 School of Mathematical Sciences, Qufu Normal University, Qufu 273165, China; \\ ${ }^{3}$ Mathematisches Institut, Albert-Ludwigs-Universität Freiburg, \\ Freiburg im Breisgau 79104, Germany.
}

Received July 2, 2019; Accepted May 25, 2020;

Published online January 18, 2021.

Dedicated to Professors Sun-Yung Alice Chang and Paul C. Yang on their 70th birthdays

\begin{abstract}
For any bounded strictly convex domain $\Omega$ in $\mathbb{R}^{n}$ with smooth boundary, we find the prescribed contact angle which is nearly perpendicular such that nonparametric mean curvature flow with contact angle boundary condition converge to ones which move by translation. Subsequently, the existence and uniqueness of smooth solutions to the capillary problem without gravity on strictly convex domain are also discussed.
\end{abstract}

AMS subject classifications: 35K59, 35J93.

Key words: Mean curvature flow, prescribed contact angle, asymptotic behavior, capillary problem.

\section{Introduction}

In this paper, we are interested in the study of the evolution of graphs defined over bounded strict convex domains $\Omega \subset \mathbb{R}^{n}$ by the nonparametric mean curvature flow, whose speed in the direction of their normal is equal to their mean curvature and with a prescribed contact angle to $\partial \Omega$.

Various results have been obtained for mean curvature flow of hypersurfaces with Dirichlet boundary conditions [26], zero-Neumann boundary condition [15], [18] and

*Corresponding author. Email addresses: gzh2333@mail .ustc.edu.cn (Z. Gao), xinan@ustc.edu.cn (X. Ma), peihewang@hotmail.com (P. Wang), ljweng08@mail.ustc.edu.cn, liangjun.weng@math.uni-freiburg.de (L. Weng) 
general Neumann boundary condition [25]. We study the evolution of graphs for $u=$ $u(x, t)$ with the speed depending on the mean curvature of the surface $\{(x, u(x, t)): x \in \Omega\}$ and with the prescribed contact angle boundary condition, that is,

$$
\begin{cases}u_{t}=\sqrt{1+|D u|^{2}} \mathcal{H}(u) & \text { in } \Omega \times(0, \infty), \\ \langle\gamma, v\rangle=\cos \theta & \text { on } \partial \Omega \times(0, \infty), \\ u(x, 0)=u_{0}(x) & \text { on } \bar{\Omega},\end{cases}
$$

where $\Omega \subset \mathbb{R}^{n}, n \geq 2$, is a compact domain with smooth boundary $\partial \Omega, \theta: \partial \Omega \rightarrow \mathbb{R}$ is the angle (contact angle) between the graph and the boundary, given by $\langle\gamma, v\rangle=\cos \theta$, which is equivalent to $u_{v}=-\cos \theta \sqrt{1+|D u|^{2}}$, where $v$ is the unit inner normal of $\partial \Omega$. Remark that one may extend $\theta$ to $\bar{\Omega}$ with $\theta \in C^{\infty}(\bar{\Omega})$. And $u_{0}(x)$ is also a smooth function satisfying the compatible condition

$$
u_{0, v}=-\cos \theta \sqrt{1+\left|D u_{0}\right|^{2}} \quad \text { on } \quad \partial \Omega
$$

While $\mathcal{H}$ is the mean curvature operator

$$
\mathcal{H}(u):=\operatorname{div}\left(\frac{D u}{\sqrt{1+|D u|^{2}}}\right)
$$

and $\gamma$ is the upward normal of the graph $\{(x, u(x, t)): x \in \Omega\}$, which is given by

$$
\gamma:=\frac{(-D u, 1)}{\sqrt{1+|D u|^{2}}}
$$

and we denote by $Q_{T}:=\Omega \times[0, T)$ for convenience.

For the prescribed contact angle boundary condition, a more general type of problem is to study the following equation, which has an extra term $F(x, u, D u)$ (some called the transport term) compared to (1.1), i.e.

$$
u_{t}=\sqrt{1+|D u|^{2}} \mathcal{H}(u)-F(x, u, D u) \quad \text { in } \quad Q_{T} .
$$

Guan [14] proved the global existence of solutions to (1.2) with prescribed contact angle condition for general bounded domain $\Omega$. Recently, Zhou generalized Guan's results to the domain $\Omega$ on Riemannian manifold in [30].

As for studying the asymptotic behavior of $u(x, t)$ in (1.2), Guan [14] or Zhou [30] only obtained the convergence results for $F(x, u, D u)$ with specific form, say $F:=\phi(x, u)$. $\sqrt{1+|D u|^{2}}$ with $\phi_{u} \geq c_{0}>0$, which excluded $F \equiv 0$. In [15], Huisken studied the fixed vertical contact angle case of (1.1), i.e. $\theta(x) \equiv \frac{\pi}{2}$, so $u_{v}=0$ on $\partial \Omega$. By using the Sobolevtype inequalities and an iteration method, Huisken proved that the solution $u(\cdot, t)$ of $(1.1)$ converges to a constant function as $t \rightarrow+\infty$. For the non-perpendicular case, Altschuler 
and $\mathrm{Wu}$ in [1] firstly considered the problem (1.1) with fixed contact angle boundary condition in one dimension, and showed that $u(x, t)$ converges to translating solitons. Subsequently, they studied in [2] for two dimension case and proved that the solutions of (1.1) converge to one which moves only by translation, under the condition that $\Omega \subset$ $\mathbb{R}^{2}$ is strictly convex and $\|D \theta\|_{C^{0}}<\min _{\partial \Omega} \kappa$, where $\kappa$ is the curvature of the curve $\partial \Omega$. The convergence results in [1] and [2] are only known now for one and two dimension cases respectively. In particular, the uniform gradient estimate is still unknown for higher space dimension. It is an open question whether the results in [1] and [2] also hold for higher space dimension? In the first part of this paper, we give a partial positive answer to this question, when the contact angle is close to $\frac{\pi}{2}$ and the domain is strictly convex.

From above discussions, we rewrite (1.1) into the following equivalent form,

$$
\begin{cases}u_{t}=\sum_{i, j=1}^{n} a_{i j} u_{i j} & \text { in } \Omega \times[0, T), \\ u_{v}=-\cos \theta(x) \sqrt{1+|D u|^{2}} & \text { on } \partial \Omega \times[0, T), \\ u(x, 0)=u_{0}(x) & \text { on } \bar{\Omega},\end{cases}
$$

where $a_{i j}:=\delta_{i j}-\frac{u_{i} u_{j}}{1+|D u|^{2}}$ and the other quantities are just the same as the ones in (1.1). Our first main result is the following convergence theorem for (1.3).

Theorem 1.1. Let $\Omega \subset \mathbb{R}^{n}(n \geq 2)$ be a strictly convex, bounded domain and $\partial \Omega \in C^{3}$. There exists $\varepsilon_{0}>0$ depending only on the convexity of $\Omega$ such that if $\theta \in C^{3}(\bar{\Omega})$ satisfies

$$
|\cos \theta| \leq \varepsilon_{0}<1, \quad \text { and } \quad\|D \theta\|_{C^{1}(\bar{\Omega})} \leq \varepsilon_{0}
$$

then the flow $u(x, t)$ in (1.3) exists for all time and converges to a translating solution to the following mean curvature equation

$$
\begin{cases}\sum_{i, j=1}^{n} a_{i j} u_{i j}=\tau & \text { in } \Omega, \\ u_{v}=-\cos \theta(x) \sqrt{1+|D u|^{2}} & \text { on } \partial \Omega .\end{cases}
$$

That is, the solution of (1.3) converges to $w(x)+\tau t$ as $t \rightarrow \infty$, which means that

$$
\lim _{t \rightarrow+\infty}\|u(\cdot, t)-(w(\cdot)+\tau t)\|_{C^{0}(\bar{\Omega})}=0,
$$

where $(\tau, w)$ is a suitable solution solving (1.5).

The crucial part of the proof is to derive an a priori estimate for the spatial gradient of $u(x, t)$, which is time-independent. This will be achieved by choosing an appropriate auxiliary function and combining with the maximum principle. Our auxiliary function and approach are motivated by methods used in [10, 24, 25, 27]. 
Remark 1.1. When $\theta(x) \equiv \frac{\pi}{2}$, Theorem 1.1 was firstly proved by Huisken in [15]. If one denotes $\theta(x):=\frac{\pi}{2}+\gamma(x)$, then condition (1.4) is equivalently reduced to: there exists $\varepsilon_{0}>0$ satisfying

$$
\|\gamma\|_{C^{2}(\bar{\Omega})} \leq \varepsilon_{0} .
$$

It is worthwhile to notice that, when the contact angle $\theta(x) \equiv \theta_{0}$ is a fixed constant, the evolution equation (1.3) is related to the so-called mean curvature flow of surface clusters, also called space partitions (networks, in the plane), see [3], [8] and references therein for more interesting physical background. As a direct corollary of Theorem 1.1, we have the following result for the fixed contact angle.

Corollary 1.1. Let $\Omega \subset \mathbb{R}^{n}(n \geq 2)$ be a strictly convex, bounded domain and $\partial \Omega \in C^{3}$. Assume that $\theta(x) \equiv \theta_{0} \in\left(0, \frac{\pi}{2}\right]$ in (1.3), then there exists $\varepsilon_{0}>0$ depending only on the convexity of $\Omega$ such that if

$$
0<\frac{\pi}{2}-\varepsilon_{0}<\theta_{0} \leq \frac{\pi}{2}
$$

the unique smooth solution $u(x, t)$ of (1.3) converges to $w(x)+\lambda t$ as $t \rightarrow \infty$, which means that

$$
\lim _{t \rightarrow+\infty}\|u(\cdot, t)-(w(\cdot)+\lambda t)\|_{C^{0}(\bar{\Omega})}=0,
$$

where $(\lambda, w)$ is a suitable solution to (1.5) with $\theta(x) \equiv \theta_{0}$ and $\tau:=\lambda$.

In fact, the existence of solutions to (1.5) is closely related to the capillary problem. Precisely, the capillary problem is referred to study the following equations.

$$
\begin{cases}\mathcal{H}(u)=\tau+k u & \text { in } \Omega, \\ \langle\gamma, v\rangle=\cos \theta & \text { on } \partial \Omega,\end{cases}
$$

where $k$ is usually referred to as the capillarity constant (see [8], Chapter 1). Results about the positive gravity $k>0$ case are extensively studied and quite well-known. Ural'tseva [29], Simon-Spruck [28] and Gerhardt [10] had obtained the existence results of (1.6) for any dimensions. More results related to positive gravity capillary problem also could be seen in [12], the wonderful exposition book by Finn in [8] and references therein. We only discuss and focus on $k=0$ (gravity free) in (1.6) in the rest part of this paper, i.e.

$$
\begin{cases}\operatorname{div}\left(\frac{D u}{\sqrt{1+|D u|^{2}}}\right)=\tau & \text { in } \Omega, \\ u_{v}=-\cos \theta \sqrt{1+|D u|^{2}} & \text { on } \partial \Omega .\end{cases}
$$

If there exists a solution to (1.7) with constant angle $\theta \equiv \theta_{0}$, integrating by parts on $\Omega$ yields that

$$
\tau=\frac{\int_{\partial \Omega} \cos \theta d \sigma}{|\Omega|}=\frac{|\partial \Omega|}{|\Omega|} \cos \theta_{0} .
$$

As pointed out by Concus and Finn in [6], Eq. (1.7) may not have any solution, even for constant angle $\theta \equiv \theta_{0} \in\left[0, \frac{\pi}{2}\right]$. In [12], Giusti proved the following results. 
Theorem 1.2 ([12]). Let $\Omega \subset \mathbb{R}^{n}(n \geq 2)$ be a Lipschitz bounded domain. If $\theta_{0} \in\left(0, \frac{\pi}{2}\right]$ and there exists $\varepsilon_{0}>0$ such that

$$
\left|\frac{|\partial \Omega|}{|\Omega|}\right| \Omega^{\prime}|-| \partial \Omega \cap \overline{\Omega^{\prime}}|| \cdot \cos \theta_{0} \leq\left(1-\varepsilon_{0}\right)\left|\partial \Omega^{\prime} \cap \Omega\right|,
$$

holds for all proper subdomains $\Omega^{\prime} \subset \Omega$. Then there exists a solution $u \in B V(\Omega)$ solving (1.7) in weak sense, with $\theta \equiv \theta_{0}$ be a fixed constant.

Nevertheless, one should note that the criterion (1.9) in Theorem 1.2 is often quite complicated and difficult to be verified, since it involves infinitely many subdomains $\Omega^{\prime} \subset$ $\Omega$. Subsequently, in 2 dimension, Giusti ( [13], Appendix) showed that (1.9) in Theorem 1.2 holds under the curvature condition $0<\kappa \leq \frac{|\partial \Omega|}{|\Omega|}$ (this implies that $\Omega$ is strictly convex), where $\kappa$ denotes the curvature of curve $\partial \Omega$. Also we mention the papers [7] and [23], where Finn and afterwards Lieberman provided another interesting viewpiont to replace criterion (1.9) with the existence of a vector field criterion. Based on those consideration and motivation, we provide below with another sufficient condition for any dimension $(n \geq 2)$, which can ensure the existence of smooth solution to (1.7). Precisely, we obtain the following result.

Theorem 1.3. Let $\Omega \subset \mathbb{R}^{n}(n \geq 2)$ be a strictly convex, bounded domain and $\partial \Omega \in C^{\infty}$. Assume $\theta \in C^{\infty}(\bar{\Omega})$, then there exists a small constant $\varepsilon_{0}>0$ only depending on the convexity of $\Omega$ such that if $\theta$ satisfies

$$
|\cos \theta| \leq \varepsilon_{0}<1, \quad \text { and }\|D \theta\|_{C^{1}(\bar{\Omega})} \leq \varepsilon_{0},
$$

then there exists a unique $\tau \in \mathbb{R}$ and a function $u \in C^{\infty}(\bar{\Omega})$ satisfying (1.7). In particular, the solution is unique up to an additive constant.

Remark 1.2. The convexity condition of the domain is necessary in the sense that FinnGiusti [9] gave an example of nonexistence for the equation (1.7) when the domain is non-convex. And if the domain is nonsmooth, there are already many works related to the generalized solution using the variational methods, see e.g. [8] (particularly Chapters 6, 7), [9], [12], and references therein.

The main difference and difficulty between the positive gravity $(k>0$ in (1.6)) and free gravity (1.7) is that there is no $C^{0}$ estimate for the solutions to (1.7), since a solution plus any constant is still a solution to (1.7). Thus one can not use the continuity method to get the existence. In order to overcome this difficulty, we use an approximation argument and obtain the uniform gradient estimate of the approximation equation, which is independent of $\|u\|_{C^{0}}$. This approach has been used previously in several different settings, see, e.g., [16, 17, 25, 27]. Those results also motivate our work here. Additionally, we want to point out that this approach is different with many former methods about the capillary problem, say e.g. $[8,12,13]$, where they usually proved the existence of generalized solutions firstly, hereafter to show that the generalized solutions possess some regularity. Here our methods are able to get the existence of smooth solution directly. 
This article is structured as follows. In Section 2, the uniform gradient estimate is established for (1.3). In Section 3, the asymptotic behavior of solution to (1.3), i.e. Theorem 1.1 is demonstrated and followed as the same as the approach used in [2], once we get the uniform gradient estimate. The last section is devoted to prove Theorem 1.3, after obtaining the uniform gradient estimate for the solutions to approximation equations.

\section{Uniform gradient estimate for mean curvature flow}

In this section, in order to study the asymptotic behavior of the nonparametric mean curvature flow with prescribed contact angle boundary condition, we establish the uniform gradient estimate for the solution to (1.3) under the condition (1.4).

We have the following facts when $\Omega$ is a strictly convex smooth domain. By the classical result (see for example Caffarelli- Nirenberg-Spruck [4] Section 2, and we can take $g^{\prime}(0)=1$ in their definition of $u$ in page 275), there exists a smooth defining function $h$ for $\Omega$ such that $h<0$ in $\Omega$ and $h=0$ on $\partial \Omega,\left\{h_{i j}\right\} \geq k_{1}\left\{\delta_{i j}\right\}$ for some constant $k_{1}>0$ and $\sup _{\Omega}|D h| \leq 1, h_{v}=-1$ and $|D h|=1$ on $\partial \Omega$. Because of the strict convexity of the domain, we may assume that the curvature matrix of $\partial \Omega$ satisfies

$$
\left\{\kappa_{i j}\right\}_{1 \leq i, j \leq n-1} \geq \kappa_{0}\left\{\delta_{i j}\right\}_{1 \leq i, j \leq n-1}
$$

for some constant $\kappa_{0}>0$. For convenience, we denote by

$$
M_{1}:=\sup _{\bar{\Omega}}\left|D^{2} h\right|, \quad M_{2}:=\sup _{\bar{\Omega}}\left|D^{3} h\right|,
$$

and define the big $O$ notation $O(s)$, which means that there exists a constant $C>0$, such that $|O(s)| \leq C$ s for $s$ large enough. In particular, we have the positive constant $C$ only depending on $M_{1}, M_{2}$ and $n$ in the rest setting of this paper.

Using the maximum principle, the same as in [2], we have a priori bound on $\left|u_{t}\right|^{2}$.

Lemma 2.1 ([2], Lemma 2.2). If $u(x, t)$ is a smooth solution to (1.3), then

$$
\sup _{Q_{T}}\left|u_{t}\right|^{2}=\left.\sup _{\Omega}\left|u_{t}\right|^{2}\right|_{t=0}
$$

holds. So there exists a constant $C=C\left(u_{0}\right)>0$ such that $\sup _{\bar{Q}_{T}}\left|u_{t}\right| \leq C$.

Next we obtain the uniform gradient estimate for (1.3), which turns the quasilinear evolution equation (1.3) into a uniformly parabolic equation and the infinite time existence of smooth solutions follows by standard regularity theory.

Theorem 2.1. Let $\Omega \subset \mathbb{R}^{n}(n \geq 2)$ be a smooth strictly convex bounded domain. There is a small constant $\varepsilon_{0}>0$ depending only on the convexity of $\Omega$ such that if $\theta \in C^{3}(\bar{\Omega})$ satisfying condition (1.4), and if $u(x, t) \in C^{3,2}\left(\bar{Q}_{T}\right)$ is a solution to (1.1), then there exists a constant $C \sim n, \Omega, u_{0}, \theta$ such that

$$
\sup _{\overline{\mathrm{Q}}_{T}}|D u| \leq C
$$


Proof. To get the uniform bound of $|D u|$ in $Q_{T}$, we only need to prove that: for $0<T^{\prime}<T$, the bound of $|D u|$ on $\bar{Q}_{T^{\prime}}$ is independent of $T^{\prime}$ and then one takes a limit argument.

Let

$$
\Phi(x, t):=\log w(x, t)+\alpha h(x),
$$

where $w(x, t):=v-\sum_{l=1}^{n} u_{l} h_{l} \cos \theta$ and $\alpha$ is a positive constant to be determined later, $v:=$ $\sqrt{1+|D u|^{2}}$.

Assume that $\Phi(x, t)$ attains the maximum value at $\left(x_{0}, t_{0}\right) \in \bar{Q}_{T^{\prime}}$. We divide it into the following three cases to complete the proof.

Case 1: $x_{0} \in \partial \Omega \times\left[0, T^{\prime}\right]$. At $x_{0}$, we choose the coordinate such that $\frac{\partial}{\partial x_{n}}$ be the inner normal direction of $\partial \Omega$, which is exactly equal to $v$, and let $\left\{x_{i}\right\}_{i=1}^{n-1}$ be the geodesic coordinate of $x_{0} \in \partial \Omega$. Along the geodesic $x_{n}=t(0<t \leq \varepsilon)$, one takes the parallel transport of tangential direction $\frac{\partial}{\partial x_{i}}$ to establish the geodesic coordinate in the neighborhood around the point $x_{0}$ in $\bar{\Omega}$. Denote $\nabla^{\prime}$ as the induced connection on $\partial \Omega$ by $D$. We denote $D_{i j}=D_{i} D_{j}$.

Firstly, we notice from boundary condition in (1.3) that

$$
w=v+u_{n} \cos \theta=v \sin ^{2} \theta \quad \text { on } \partial \Omega .
$$

We denote $\nabla^{\prime} u$ and $u_{n}$ as the tangential and normal part of $D u$ on the boundary by our choice of coordinate above. We also denote $\nabla_{i}^{\prime}\left(u_{n}\right):=u_{n i}$. From the boundary condition $u_{n}=-v \cos \theta$, we deduce that

$$
u_{n}^{2}=v^{2} \cos ^{2} \theta=\cos ^{2} \theta\left(1+\left|\nabla^{\prime} u\right|^{2}+u_{n}^{2}\right),
$$

so it directly follows that

$$
u_{n}^{2}=\cot ^{2} \theta\left(1+\left|\nabla^{\prime} u\right|^{2}\right),
$$

and in particular, we have

$$
w=v \sin ^{2} \theta=\sqrt{1+\left|\nabla^{\prime} u\right|^{2}+u_{n}^{2}} \sin ^{2} \theta=\sqrt{1+\left|\nabla^{\prime} u\right|^{2}} \sin \theta,
$$

and

$$
v w=1+\left|\nabla^{\prime} u\right|^{2} .
$$

From Gauss-Weingarten equation and directly computation, we have

$$
\begin{aligned}
D_{n} v & =\frac{1}{v} \sum_{k=1}^{n} u_{k} D_{k n} u=\frac{1}{v} \sum_{i=1}^{n-1} u_{i} D_{i n} u-\cos \theta D_{n n} u \\
& =\frac{1}{v} \sum_{i=1}^{n-1}\left(u_{i} u_{n i}+\sum_{j=1}^{n-1} u_{i} b_{i j} u_{j}\right)-\cos \theta D_{n n} u,
\end{aligned}
$$


where $b_{i j}$ is the second fundamental form of $\partial \Omega$. Then at $x_{0} \in \partial \Omega$, it follows that

$$
\begin{aligned}
0 & \geq D_{n} \Phi\left(x_{0}\right)=\frac{D_{n} w}{w}+\alpha h_{n} \\
& =\frac{1}{w}\left[D_{n} v-D_{n}\left(\sum_{k=1}^{n} u_{k} h_{k}\right) \cos \theta-D_{n}(\cos \theta) \cdot \sum_{k=1}^{n} u_{k} h_{k}\right]-\alpha \\
& =\frac{1}{w}\left[D_{n} v-\sum_{k=1}^{n} D_{n k} u h_{k} \cos \theta-\sum_{k=1}^{n} u_{k} D_{n k} h \cos \theta+\sum_{k=1}^{n} u_{k} h_{k} \sin \theta \theta_{n}\right]-\alpha \\
& =\frac{1}{w}\left[D_{n} v+D_{n n} u \cos \theta-\sum_{k=1}^{n} u_{k} D_{n k} h \cos \theta-\sin \theta \theta_{n} u_{n}\right]-\alpha \\
& =\frac{1}{v w} \sum_{i=1}^{n-1}\left(u_{i} u_{n i}+\sum_{j=1}^{n-1} b_{i j} u_{i} u_{j}\right)-\frac{1}{w} \sum_{k=1}^{n} u_{k} D_{n k} h \cos \theta+\cot \theta \theta_{n}-\alpha .
\end{aligned}
$$

And for all $1 \leq i \leq n-1$, since $\left\{x_{i}\right\}$ is the tangential direction, we obtain

$$
0=\nabla_{i}^{\prime} \Phi\left(x_{0}\right)=\frac{\nabla_{i}^{\prime} w}{w}=\frac{1}{w}\left[\nabla_{i}^{\prime} v+u_{n i} \cos \theta-u_{n} \sin \theta \nabla_{i}^{\prime} \theta\right]
$$

this implies that

$$
\nabla_{i}^{\prime} v=-u_{n i} \cos \theta+u_{n} \sin \theta \nabla_{i}^{\prime} \theta .
$$

On the other hand, by taking the tangential derivative to the boundary condition of (1.3) and combining with (2.4), it yields that

$$
u_{n i}=\nabla_{i}^{\prime}(-\cos \theta v)=-\cos \theta \nabla_{i}^{\prime} v+\sin \theta \nabla_{i}^{\prime} \theta v=\cos ^{2} \theta u_{n i}-\cos \theta \sin \theta u_{n} \nabla_{i}^{\prime} \theta+\sin \theta \nabla_{i}^{\prime} \theta v,
$$

then it follows that

$$
u_{n i}=(\cos \theta \cot \theta+\csc \theta) \nabla_{i}^{\prime} \theta v, \quad \text { for } i=1, \cdots, n .
$$

Hence, we get

$$
\begin{aligned}
& \frac{1}{v w} \sum_{i=1}^{n-1}\left(u_{i} u_{n i}+\sum_{j=1}^{n-1} b_{i j} u_{i} u_{j}\right)=\frac{1}{v w} \sum_{i, j=1}^{n-1} b_{i j} u_{i} u_{j}+\frac{1}{w}(\cos \theta \cot \theta+\csc \theta) \sum_{i=1}^{n-1} u_{i} \nabla_{i}^{\prime} \theta \\
& -\frac{1}{w} \sum_{k=1}^{n} u_{k} D_{n k} h \cos \theta=-\frac{1}{w}\left(\sum_{i=1}^{n-1} u_{i} D_{n i} h+u_{n} D_{n n} h\right) \cos \theta
\end{aligned}
$$

Substituting Eqs. (2.5) and (2.6) into (2.3), and note that we have condition (1.4) with 
$\varepsilon_{0}<1$, thus we have

$$
\begin{aligned}
& 0 \geq D_{n} \Phi\left(x_{0}\right)=\frac{1}{v w} \sum_{i, j=1}^{n-1} b_{i j} u_{i} u_{j}+\frac{1}{w}(\cos \theta \cot \theta+\csc \theta) \sum_{i=1}^{n-1} u_{i} \theta_{i} \\
& -\frac{1}{w}\left(\sum_{i=1}^{n-1} u_{i} D_{n i} h+u_{n} D_{n n} h\right) \cos \theta+\cot \theta \theta_{n}-\alpha \\
& \geq \frac{\kappa_{0}}{v w}\left|\nabla^{\prime} u\right|^{2}-\left(\cot ^{2} \theta+\csc ^{2} \theta\right) \frac{\left|\nabla^{\prime} u\right|}{\sqrt{1+\left|\nabla^{\prime} u\right|^{2}}}\left|\nabla^{\prime} \theta\right|-\frac{\left|\nabla^{\prime} u\right|}{\sqrt{1+\left|\nabla^{\prime} u\right|^{2}}}|\cot \theta| M_{1} \\
& +\cot ^{2} \theta k_{1}+\cot \theta \theta_{n}-\alpha \\
& \geq \kappa_{0} \cdot \frac{\left|\nabla^{\prime} u\right|^{2}}{1+\left|\nabla^{\prime} u\right|^{2}}-\frac{2}{\sin ^{2} \theta}\left|\nabla^{\prime} \theta\right|-\left(M_{1}+|D \theta|\right)|\cot \theta|-\alpha \\
& \geq \kappa_{0} \cdot \frac{\left|\nabla^{\prime} u\right|^{2}}{1+\left|\nabla^{\prime} u\right|^{2}}-\frac{\varepsilon_{0}}{1-\varepsilon_{0}^{2}}\left(3+M_{1}\right)-\alpha,
\end{aligned}
$$

where $M_{1}:=\sup _{\bar{\Omega}}\left|D^{2} h\right|$. Then it yields that

$$
\left[\kappa_{0}-\frac{\varepsilon_{0}}{1-\varepsilon_{0}^{2}}\left(M_{1}+3\right)-\alpha\right]\left|\nabla^{\prime} u\right|^{2} \leq \frac{\varepsilon_{0}}{1-\varepsilon_{0}^{2}}\left(M_{1}+3\right)+\alpha .
$$

By choosing $\alpha, \varepsilon_{0}>0$ such that

$$
0<\alpha \leq \frac{\kappa_{0}}{3}, \quad \text { and any } \quad \varepsilon_{0} \in\left(0, \frac{\kappa_{0}}{9\left(M_{1}+3\right)}\right],
$$

it follows from (2.7) that we have $\left|\nabla^{\prime} u\right|^{2} \leq 2$, so the estimate of $|D u|$ follows by combining this with equation (2.2).

Case 2: $x_{0} \in \Omega$ and $t_{0}=0$, then we have

$$
\Phi(x, t) \leq \Phi\left(x_{0}, 0\right)=\log \left(\sqrt{1+\left|D u_{0}\right|^{2}}-\left\langle D u_{0}, D h\right\rangle \cos \theta\right)+\alpha h \leq C\left(u_{0}, \sup _{\Omega}|h|, \alpha\right) .
$$

Since $|\cos \theta| \leq \varepsilon_{0}<1$, it yields from above that

$$
\sup _{\Omega_{T}} v \leq C\left(u_{0}, \sup _{\Omega}|h|\right) .
$$

Case 3: $x_{0} \in \Omega$ and $T^{\prime} \geq t_{0}>0$, so at $\left(x_{0}, t_{0}\right)$, we have

$$
0=\Phi_{i}\left(x_{0}, t_{0}\right)=\frac{w_{i}}{w}+\alpha h_{i},
$$

and

$$
\begin{aligned}
0 & \geq \sum_{i, j=1}^{n} a_{i j} \Phi_{i j}\left(x_{0}, t_{0}\right)-\Phi_{t}\left(x_{0}, t_{0}\right) \\
& =\left(\sum_{i, j=1}^{n} \frac{a_{i j} w_{i j}}{w}-\frac{w_{t}}{w}\right)-\alpha^{2} \sum_{i, j=1}^{n} a_{i j} h_{i} h_{j}+\alpha \sum_{i, j=1}^{n} a_{i j} h_{i j}=: \mathrm{I}+\mathrm{II}+\mathrm{III} .
\end{aligned}
$$


We choose a proper coordinates at $\left(x_{0}, t_{0}\right)$ such that $|D u|\left(x_{0}, t_{0}\right)=u_{1}\left(x_{0}, t_{0}\right)$ and $\left\{u_{i j}\left(x_{0}, t_{0}\right)\right\}_{2 \leq i, j \leq n}$ is diagonal. Then at $\left(x_{0}, t_{0}\right)$,

$$
a_{11}=\frac{1}{v^{2}}, a_{i j}=0 \quad \text { for } i \neq j \text { and } a_{i i}=1 \text { for } i \geq 2
$$

We always assume that $u_{1}\left(x_{0}, t_{0}\right)$ is large enough in the below computation, such that $u_{1}, v=\sqrt{1+u_{1}^{2}}$, and $w=v-u_{1} h_{1} \cos \theta$ (since we assume $|\cos \theta| \leq \varepsilon_{0}<1$ ) are comparable at $\left(x_{0}, t_{0}\right)$, that is, if we let $u_{1} \geq 1$ (otherwise $\left|u_{1}\right|$ is bounded by 1 ), then

$$
u_{1} \leq v \leq \sqrt{2} u_{1}, \quad\left(1-\varepsilon_{0}\right) u_{1} \leq w \leq\left(\sqrt{2}+\varepsilon_{0}\right) u_{1}, \quad\left(1-\varepsilon_{0}\right) v \leq w \leq\left(1+\varepsilon_{0}\right) v .
$$

All the computation below will be done at the point $\left(x_{0}, t_{0}\right)$. We have

$$
\mathrm{II}=-\alpha^{2} \sum_{i, j=1}^{n} a_{i j} h_{i} h_{j}=-\alpha^{2}\left(\frac{h_{1}^{2}}{v^{2}}+\sum_{i=2}^{n} h_{i}^{2}\right) \geq-\alpha^{2}\left[\frac{1}{v^{2}}+1\right],
$$

and

$$
\mathrm{III}=\alpha \sum_{i, j=1}^{n} a_{i j} h_{i j}=\alpha\left(\frac{h_{11}}{v^{2}}+\sum_{i=2}^{n} h_{i i}\right) \geq \alpha k_{1}\left[\frac{1}{v^{2}}+(n-1)\right] .
$$

We denote by $\mathrm{J}:=\sum_{i, j=1}^{n} a_{i j} w_{i j}-w_{t}$. From (2.10), we have

$$
\sum_{l=1}^{n}\left(\frac{u_{l} u_{l i}}{v}-u_{l i} h_{l} \cos \theta-u_{l} h_{l i} \cos \theta+u_{l} h_{l} \sin \theta \theta_{i}\right)=-\alpha h_{i} w .
$$

If we denote by

$$
\mathrm{S}_{l}:=\frac{u_{l}}{v}-h_{l} \cos \theta, \quad \text { for } l=1, \cdots, n,
$$

then we have the bound as $2 \geq S_{1} \geq \frac{1}{4}$ if we assume $u_{1}^{2} \geq 1$ and $\varepsilon_{0} \leq \frac{1}{4}$. Hence, then we obtain

$$
\sum_{l=1}^{n} \mathrm{~S}_{l} u_{l i}=h_{1 i} \cos \theta u_{1}-h_{1} \sin \theta \theta_{i} u_{1}-\alpha h_{i} w
$$

It follows that for $i=2, \cdots, n$,

$$
\begin{aligned}
u_{1 i} & =-\frac{\mathrm{S}_{i}}{\mathrm{~S}_{1}} u_{i i}+\frac{h_{1 i} \cos \theta}{\mathrm{S}_{1}} u_{1}-\frac{h_{1} \sin \theta \theta_{i}}{\mathrm{~S}_{1}} u_{1}-\frac{\alpha h_{i}}{\mathrm{~S}_{1}} w \\
& =-\frac{\mathrm{S}_{i}}{\mathrm{~S}_{1}} u_{i i}+O(|\cos \theta|+|D \theta|) u_{1}+O(\alpha) w,
\end{aligned}
$$


and

$$
\begin{aligned}
u_{11} & =-\sum_{k=2}^{n} \frac{S_{k}}{S_{1}} u_{1 k}+\frac{h_{11} \cos \theta}{S_{1}} u_{1}-\frac{h_{1} \sin \theta \theta_{1}}{S_{1}} u_{1}-\frac{\alpha h_{1}}{S_{1}} w \\
& =\sum_{k=2}^{n} \frac{S_{k}^{2}}{S_{1}^{2}} u_{k k}+O(|\cos \theta|+|D \theta|) u_{1}+O(\alpha) w .
\end{aligned}
$$

To handle the term I, we take the first derivative with respect to $x_{k}$ to the equation in (1.3),

$$
u_{k t}=\left(\sum_{i, j=1}^{n} a_{i j} u_{i j}\right)_{k}=\sum_{i, j=1}^{n} a_{i j, k} u_{i j}+\sum_{i, j=1}^{n} a_{i j} u_{i j k} .
$$

By direct computation, we have

$$
\begin{aligned}
w_{t} & =v_{t}-\left(\sum_{l=1}^{n} u_{l} h_{l} \cos \theta\right)_{t}=\sum_{l=1}^{n}\left(\frac{u_{l} u_{l t}}{v}-u_{l t} h_{l} \cos \theta\right)=\sum_{l=1}^{n} \mathrm{~S}_{l} u_{l t} \\
& =\sum_{k=1}^{n} \mathrm{~S}_{k}\left[\sum_{i, j=1}^{n} a_{i j, k} u_{i j}+\sum_{i, j=1}^{n} a_{i j} u_{i j k}\right] .
\end{aligned}
$$

and

$$
\begin{aligned}
w_{i j}= & v_{i j}-\sum_{k=1}^{n}\left(u_{k} h_{k} \cos \theta\right)_{i j} \\
= & \sum_{k=1}^{n} \frac{u_{k i} u_{k j}}{v}+\sum_{k=1}^{n} \frac{u_{k} u_{k i j}}{v}-\sum_{k, l=1}^{n} \frac{u_{k} u_{k i} u_{l} u_{l j}}{v^{3}}-\sum_{l=1}^{n} u_{l i} h_{l j} \cos \theta-\sum_{l=1}^{n} u_{l j} h_{l i} \cos \theta-u_{1} h_{1 i j} \cos \theta \\
& -\sum_{l=1}^{n} u_{l i j} h_{l} \cos \theta-\sum_{k=1}^{n}\left(u_{k} h_{k}\right)_{i}(\cos \theta)_{j}-\sum_{k=1}^{n}\left(u_{k} h_{k}\right)_{j}(\cos \theta)_{i}-\sum_{k=1}^{n} u_{k} h_{k}(\cos \theta)_{i j}
\end{aligned}
$$

By (2.17) and (2.18), thus it follows that

$$
\begin{aligned}
& \mathrm{J}=w \mathrm{I}=\sum_{i, j=1}^{n} a_{i j} w_{i j}-w_{t} \\
& =\sum_{i, j=1}^{n} a_{i j}\left(\sum_{k=1}^{n} \frac{u_{k i} u_{k j}}{v}-\sum_{k, l=1}^{n} \frac{u_{k} u_{k i} u_{l} u_{l j}}{v^{3}}\right) \\
& \quad+\sum_{i, j, k=1}^{n}\left[a_{i j}\left(\frac{u_{k} u_{k i j}}{v}-u_{k i j} h_{k} \cos \theta\right)-S_{k} a_{i j, k} u_{i j}-S_{k} a_{i j} u_{i j k}\right] \\
& \quad-\sum_{i, j, k=1}^{n} a_{i j} u_{k}\left(h_{k i j} \cos \theta-2 h_{k i} \sin \theta \theta_{j}-h_{k} \cos \theta \theta_{i} \theta_{j}-h_{k} \sin \theta \theta_{i j}\right) \\
& \quad-2 \sum_{i, j, k=1}^{n} a_{i j} u_{k i}\left(h_{k j} \cos \theta-h_{k} \sin \theta \theta_{j}\right) \\
& =: \mathrm{J}_{1}+\mathrm{J}_{2}+\mathrm{J}_{3}+\mathrm{J}_{4} .
\end{aligned}
$$


We can derive that

$$
\begin{aligned}
\mathrm{J}_{3}= & -\sum_{i, j, k=1}^{n} a_{i j} u_{k}\left(h_{k i j} \cos \theta-2 h_{k i} \sin \theta \theta_{j}-h_{k} \cos \theta \theta_{i} \theta_{j}-h_{k} \sin \theta \theta_{i j}\right) \\
= & -h_{111} \cos \theta \frac{u_{1}}{v^{2}}-\sum_{i=2}^{n} h_{1 i i} \cos \theta u_{1}+2 h_{11} \sin \theta \theta_{1} \frac{u_{1}}{v^{2}}+2 \sum_{i=2}^{n} h_{1 i} \sin \theta \theta_{i} u_{1} \\
& +h_{1} \cos \theta \theta_{1}^{2} \frac{u_{1}}{v^{2}}+\sum_{i=2}^{n} h_{1} \cos \theta \theta_{i}^{2} u_{1}+h_{1} \sin \theta \theta_{11} \frac{u_{1}}{v^{2}}+\sum_{i=2}^{n} h_{1} \sin \theta \theta_{i i} u_{1} \\
\geq & -C\left(|\cos \theta|+|D \theta|+\left|D^{2} \theta\right|\right) u_{1} .
\end{aligned}
$$

Using (2.15) and (2.16), we get

$$
\begin{aligned}
\mathrm{J}_{4}= & -2 \sum_{i, j, k=1}^{n} a_{i j} u_{k i}\left(h_{k j} \cos \theta-h_{k} \sin \theta \theta_{j}\right) \\
=- & 2 \sum_{k=1}^{n}\left(h_{k 1} \cos \theta-h_{k} \sin \theta \theta_{1}\right) \frac{u_{1 k}}{v^{2}}-2 \sum_{k=1}^{n} \sum_{i=2}^{n}\left(h_{k i} \cos \theta-h_{k} \sin \theta \theta_{i}\right) u_{k i} \\
= & -2\left(h_{11} \cos \theta-h_{1} \sin \theta \theta_{1}\right) \frac{u_{11}}{v^{2}}-2 \sum_{k=2}^{n}\left(h_{k 1} \cos \theta-h_{k} \sin \theta \theta_{1}\right) \frac{u_{1 k}}{v^{2}} \\
& -2 \sum_{i=2}^{n}\left(h_{1 i} \cos \theta-h_{1} \sin \theta \theta_{i}\right) u_{1 i}-2 \sum_{i=2}^{n}\left(h_{i i} \cos \theta-h_{i} \sin \theta \theta_{i}\right) u_{i i} \\
=- & 2\left(h_{11} \cos \theta-h_{1} \sin \theta \theta_{1}\right)\left[\sum_{k=2}^{n} \frac{S_{k}^{2}}{S_{1}^{2}} u_{k k}+O(|\cos \theta|+|D \theta|) u_{1}+O(\alpha) w\right] \frac{1}{v^{2}} \\
& -2 \sum_{k=2}^{n}\left(h_{k 1} \cos \theta-h_{k} \sin \theta \theta_{1}\right)\left[-\frac{S_{k}}{S_{1}} u_{k k}+O(|\cos \theta|+|D \theta|) u_{1}+O(\alpha) w\right] \frac{1}{v^{2}} \\
& -2 \sum_{i=2}^{n}\left(h_{1 i} \cos \theta-h_{1} \sin \theta \theta_{i}\right)\left[-\frac{S_{i}}{S_{1}} u_{i i}+O(|\cos \theta|+|D \theta|) u_{1}+O(\alpha) w\right] \\
& -2 \sum_{i=2}^{n}\left(h_{i i} \cos \theta-h_{i} \sin \theta \theta_{i}\right) u_{i i} \\
\geq & C(|\cos \theta|+|D \theta|)\left(\sum_{i=2}^{n}\left|u_{i i}\right|+u_{1}\right) .
\end{aligned}
$$

It follows that

$$
\mathrm{J}_{3}+\mathrm{J}_{4} \geq-C\left(|\cos \theta|+|D \theta|+\left|D^{2} \theta\right|\right) u_{1}-C(|\cos \theta|+|D \theta|) \sum_{i=2}^{n}\left|u_{i i}\right| .
$$


A direct computation gives

$$
\begin{aligned}
\mathrm{J}_{2} & =\sum_{i, j, k=1}^{n}\left[a_{i j}\left(\frac{u_{k} u_{k i j}}{v}-u_{k i j} h_{k} \cos \theta\right)-\mathrm{S}_{k} a_{i j, k} u_{i j}-\mathrm{S}_{k} a_{i j} u_{i j k}\right] \\
& =-\sum_{i, j, k=1}^{n} \mathrm{~S}_{k} a_{i j, k} u_{i j}=-\sum_{i, j, k=1}^{n} \mathrm{~S}_{k} u_{i j}\left(-2 \frac{u_{i k} u_{j}}{v^{2}}+2 \sum_{l=1}^{n} \frac{u_{i} u_{j} u_{l k} u_{l}}{v^{4}}\right) \\
& =2 \frac{1}{v^{2}} \sum_{i=1}^{n} u_{1} u_{1 i}\left(\sum_{l=1}^{n} \mathrm{~S}_{l} u_{i l}\right)-\frac{2}{v^{4}} u_{1}^{3} u_{11} \sum_{l=1}^{n} \mathrm{~S}_{l} u_{1 l} \\
& =2 \frac{u_{1} u_{11}}{v^{4}} \sum_{l=1}^{n} \mathrm{~S}_{l} u_{1 l}+2 \sum_{i=2}^{n} u_{1 i} \frac{u_{1}}{v^{2}}\left(\sum_{l=1}^{n} \mathrm{~S}_{l} u_{i l}\right) \\
& =\frac{2 u_{1} u_{11}}{v^{4}}\left[\mathrm{~S}_{1} u_{11}+\sum_{l=2}^{n} \mathrm{~S}_{l} u_{1 l}\right]+\frac{2 u_{1}}{v^{2}}\left[\mathrm{~S}_{1} \sum_{i=2}^{n} u_{1 i}^{2}+\sum_{i=2}^{n} \mathrm{~S}_{i} u_{i i} u_{1 i}\right] \\
& =\frac{2 u_{1} \mathrm{~S}_{1}}{v^{4}} u_{11}^{2}+\frac{2 u_{1} u_{11}}{v^{4}} \sum_{l=2}^{n} \mathrm{~S}_{l} u_{1 l}+\frac{2 u_{1} \mathrm{~S}_{1}}{v^{2}} \sum_{i=2}^{n} u_{1 i}^{2}+\frac{2 u_{1}}{v^{2}} \sum_{i=2}^{n} \mathrm{~S}_{i} u_{i i} u_{1 i} .
\end{aligned}
$$

By expanding the sum in $\mathrm{J}_{1}$, it is easy to obtain that

$$
\mathrm{J}_{1}=\frac{1}{v^{5}} u_{11}^{2}+\frac{2}{v^{3}} \sum_{i=2}^{n} u_{1 i}^{2}+\frac{1}{v} \sum_{i=2}^{n} u_{i i}^{2}
$$

So we have

$$
\begin{aligned}
\mathrm{J}_{1}+\mathrm{J}_{2}=\left[\frac{1}{v^{5}}\right. & \left.+\frac{2 u_{1} \mathrm{~S}_{1}}{v^{4}}\right] u_{11}^{2}+\left[\frac{2}{v^{3}}+\frac{2 u_{1} \mathrm{~S}_{1}}{v^{2}}\right] \sum_{i=2}^{n} u_{1 i}^{2} \\
& +\frac{1}{v} \sum_{i=2}^{n} u_{i i}^{2}+\frac{2 u_{1} u_{11}}{v^{4}} \sum_{l=2}^{n} \mathrm{~S}_{l} u_{1 l}+\frac{2 u_{1}}{v^{2}} \sum_{i=2}^{n} \mathrm{~S}_{i} u_{i i} u_{1 i} .
\end{aligned}
$$

From $2 \geq \mathrm{S}_{1} \geq \frac{1}{4}$ and for $2 \leq i \leq n,\left|\mathrm{~S}_{i}\right| \leq|\cos \theta|$, we can use the Cauchy inequality

$$
\begin{aligned}
& \left|\frac{2 u_{1} u_{11}}{v^{4}} \sum_{l=2}^{n} S_{l} u_{1 l}\right| \leq \frac{2 u_{1} S_{1}}{v^{4}} u_{11}^{2}+\frac{(n-1)|\cos \theta|^{2} u_{1}}{2 S_{1} v^{4}} \sum_{i=2}^{n} u_{1 i}^{2}, \\
& \left|\frac{2 u_{1}}{v^{2}} \sum_{i=2}^{n} S_{i} u_{i i} u_{1 i}\right| \leq \frac{u_{1} S_{1}}{v^{2}} \sum_{i=2}^{n} u_{1 i}^{2}+\frac{u_{1}|\cos \theta|^{2}}{S_{1} v^{2}} \sum_{i=2}^{n} u_{i i}^{2} .
\end{aligned}
$$

Substituting (2.26) and (2.27) into (2.25), if we assume $|\cos \theta| \leq \frac{1}{4}$, we obtain

$$
\mathrm{J}_{1}+\mathrm{J}_{2} \geq \frac{1}{2 v} \sum_{i=2}^{n} u_{i i}^{2}
$$


Substituting (2.19), (2.22) and (2.28), we get

$$
\begin{aligned}
\mathrm{J} & \geq \frac{1}{2 v} \sum_{i=2}^{n} u_{i i}^{2}-C(|\cos \theta|+|D \theta|) \sum_{i=2}^{n}\left|u_{i i}\right|-C\left(|\cos \theta|+|D \theta|+\left|D^{2} \theta\right|\right) u_{1} \\
& \geq \frac{1}{4 v} \sum_{i=2}^{n} u_{i i}^{2}-C\left(|\cos \theta|+|D \theta|+\left|D^{2} \theta\right|\right) v .
\end{aligned}
$$

Hence, finally we derive that

$$
\mathrm{I}=\frac{\mathrm{J}}{w} \geq-C\left(|\cos \theta|+|D \theta|+\left|D^{2} \theta\right|\right) .
$$

Combining this with (2.11)-(2.13) together, we obtain

$$
0 \geq \mathrm{I}+\mathrm{II}+\mathrm{III} \geq-C\left(|\cos \theta|+|D \theta|+\left|D^{2} \theta\right|\right)-\alpha^{2}\left(\frac{1}{v^{2}}+1\right)+\alpha k_{1}\left(\frac{1}{v^{2}}+(n-1)\right) .
$$

By taking $\alpha:=\min \left\{\frac{k_{1}}{2}, \frac{\kappa_{0}}{3}, 1\right\}:=\alpha_{0}$ and $\varepsilon_{0}=\min \left\{\frac{\kappa_{0}}{9\left(M_{1}+3\right)}, \frac{\alpha_{0} k_{1}}{2 C_{1}}, \frac{1}{4}\right\}$ in (1.4), we obtain

$$
v\left(x_{0}, t_{0}\right) \leq \tilde{C},
$$

where $\tilde{C}$ is independent of $T^{\prime}$.

Combining three cases above together, we get the uniform estimate for $|D u|$ which is independent of $T^{\prime}$ and then Theorem 2.1 is proved.

\section{Elliptic interlude and asymptotic behavior}

As the approach in the two dimension case in the paper [2], our gradient estimate also can be used to solve the elliptic version of the problem. The elliptic version of equation (1.3) is

$$
\begin{cases}\sum_{i, j=1}^{n}\left(\delta_{i j}-\frac{u_{i} u_{j}}{1+|D u|^{2}}\right) u_{i j}=\tau & \text { in } \Omega, \\ u_{v}=-\cos \theta \sqrt{1+|D u|^{2}} & \text { on } \partial \Omega,\end{cases}
$$

where $\tau \in \mathbb{R}$ is a uniquely determined constant. In fact, by using the integration by parts, one can see that

$$
\tau=\frac{\int_{\partial \Omega} \cos \theta d \sigma}{\int_{\Omega}\left(1+|D u|^{2}\right)^{-\frac{1}{2}} d x} .
$$

We can obtain the following existence result for (3.1) in high space dimension case under the condition (1.4). For 2 dimension, this result was proved by Altschuler-Wu (see Theorem 2.6 in [2]) under more generally condition on $\Omega$ and $\theta$. 
Theorem 3.1. Let $\Omega \subset \mathbb{R}^{n}$ ( $n \geq 3$ ) be a smooth strictly convex bounded domain. There is a small constant $\varepsilon_{0}>0$ such that if $\theta \in C^{3}(\bar{\Omega})$ satisfying condition (1.4), then there exists a unique $\tau$ and a smooth solution $u$ for (3.1). In particular, the solution $u$ is unique up to an additive constant.

Proof. Firstly one wants to consider the following problem,

$$
\begin{cases}\sum_{i, j=1}^{n}\left(\delta_{i j}-\frac{u_{i} u_{j}}{1+|D u|^{2}}\right) u_{i j}=\varepsilon u & \text { in } \Omega, \\ u_{v}=-\cos \theta \sqrt{1+|D u|^{2}} & \text { on } \quad \partial \Omega .\end{cases}
$$

Using the barrier argument in [2] (see the proof of Theorem 2.6), one obtains $\sup _{\bar{\Omega}}|\varepsilon u| \leq$ $C_{0}$, where $C_{0}$ is independent of $\varepsilon$. Under the condition (1.4), we want to show the solution to (3.3) has the uniform gradient estimate, which is independent of $\varepsilon$, i.e. $|D u| \leq C$. This can be proved by the same procedure as the proof of Theorem 2.1 by now replacing $u_{t}$ in (1.3) with $\varepsilon$ u.

We choose the same $\Phi(x):=\log w(x)+\alpha h(x)$ and the boundary case is treated by the Case 1 in Section 2. Now we assume the $\Phi(x)$ attains its maximum at $x_{0} \in \Omega$.

So at $x_{0}$, we have $0=\Phi_{i}\left(x_{0}\right)=\frac{w_{i}}{w}+\alpha h_{i}$ and

$$
0 \geq \sum_{i, j=1}^{n} a_{i j} \Phi_{i j}\left(x_{0}\right)=\sum_{i, j=1}^{n} \frac{a_{i j} w_{i j}}{w}-\alpha^{2} \sum_{i, j=1}^{n} a_{i j} h_{i} h_{j}+\alpha \sum_{i, j=1}^{n} a_{i j} h_{i j}=: \mathrm{I}+\mathrm{II}+\mathrm{III} .
$$

We choose a proper coordinates at $x_{0}$ such that $|D u|\left(x_{0}\right)=u_{1}\left(x_{0}\right)$ and $\left\{u_{i j}\left(x_{0}\right)\right\}_{2 \leq i, j \leq n}$ is diagonal. Then at $x_{0}$,

$$
\mathrm{II}+\mathrm{III} \geq-\alpha^{2}\left(\frac{1}{v^{2}}+1\right)+\alpha k_{1}\left(\frac{1}{v^{2}}+(n-1)\right) .
$$

We denote by $\mathrm{J}:=\sum_{i, j=1}^{n} a_{i j} w_{i j}$ and as in (2.19), it follows that

$$
\mathrm{J}=w \mathrm{I}:=\mathrm{J}_{1}+\widetilde{J}_{2}+\mathrm{J}_{3}+\mathrm{J}_{4},
$$

where $\mathrm{J}_{1}, \mathrm{~J}_{3}, \mathrm{~J}_{4}$ are defined as in (2.19). Using Eq. (3.3) we have

$$
\begin{aligned}
\widetilde{J}_{2} & :=\sum_{i, j, k=1}^{n} a_{i j}\left(\frac{u_{k} u_{k i j}}{v}-u_{k i j} h_{k} \cos \theta\right)=\sum_{i, j, k=1}^{n} \mathrm{~S}_{k} a_{i j} u_{i j k} \\
& =-\sum_{i, j, k=1}^{n} \mathrm{~S}_{k} a_{i j, k} u_{i j}+\sum_{k=1}^{n} \mathrm{~S}_{k}(\varepsilon u)_{k}=\mathrm{J}_{2}+\varepsilon u_{1} \mathrm{~S}_{1} \geq \mathrm{J}_{2} .
\end{aligned}
$$

By the same procedure as the proof of Theorem 2.1 we can get the the uniform gradient estimates which is independent of $\varepsilon$.

From the $C^{0}$ and $C^{1}$ estimates, we get the existence of the solution to (3.3) by standard theory in [11] for each $\varepsilon>0$. 
The uniform estimate of $|D u|$ also implies that $|D(\varepsilon u)| \rightarrow 0$ as $\varepsilon \rightarrow 0$. Thus one can conclude that $\varepsilon \mathcal{u} \rightarrow \tau$ as $\varepsilon \rightarrow 0$ for some $\tau \in \mathbb{R}$ (One can see the detail proof on the existence part for the similar limited equation in Theorem 1.3 ).

To show the uniqueness, as in [2] (see the proof of Theorem 2.6 there) one assumes that if $\left(u_{1}, \tau_{1}\right)$ and $\left(u_{2}, \tau_{2}\right)$ are two solutions to (3.1). Without loss of generality, we assume $\tau_{1} \leq \tau_{2}$, denote $u:=u_{1}-u_{2}$. By direct computation, we obtain that $u$ is the super-solution of the following elliptic operator

$$
L u:=\sum_{i, j=1}^{n} \hat{a}_{i j} u_{i j}+\sum_{l=1}^{n} b_{l} u_{l}
$$

where

$$
\hat{a}_{i j}:=a_{i j}\left(D u_{1}\right), \quad b_{l}:=\sum_{i, j=1}^{n} \int_{0}^{1} a_{i j, p_{l}}\left(t D u_{1}+(1-t) D u_{2}\right) d t \cdot\left(u_{2}\right)_{i j} .
$$

From the maximum principle, $u$ attains the maximum value on the boundary, say at $x_{0} \in \partial \Omega$. Thus combining with Hopf lemma, we have $\nabla^{\prime} u\left(x_{0}\right)=0$ and $D_{v} u\left(x_{0}\right)<0$, that is, $\left|\nabla^{\prime} u_{1}\right|=\left|\nabla^{\prime} u_{2}\right|=q$ and $D_{v} u_{1}<D_{v} u_{2}$, where we denote $\nabla^{\prime}$ and $D_{v}$ as the tangential and normal part of $D$ on boundary respectively. On the other hand, from the boundary condition in (3.1), it follows that

$$
\frac{D_{v} u_{1}}{\sqrt{1+q^{2}+\left|D_{v} u_{1}\right|^{2}}}=\frac{D_{v} u_{2}}{\sqrt{1+q^{2}+\left|D_{v} u_{2}\right|^{2}}}
$$

but this is a contradiction with the fact that function $\frac{x}{\sqrt{1+q^{2}+x^{2}}}$ is strictly increasing with respect to $x \in \mathbb{R}$ and $D_{v} u_{1}\left(x_{0}\right)<D_{v} u_{2}\left(x_{0}\right)$. So $u$ must be a constant, and $\tau_{1}=\tau_{2}$. Therefore, we have completed the proof.

Remark 3.1. For any $\varepsilon>0$, even for any bounded smooth domain, the existence of solution to (3.3) could be got from the standard method on the capillary problem with positive gravity, see for example Theorem 9.12 in Lieberman [22] or [19].

Remark 3.2. If one considers the following problem,

$$
\begin{cases}\sum_{i, j=1}^{n}\left(\delta_{i j}-\frac{u_{i} u_{j}}{1+|D u|^{2}}\right) u_{i j}=\varepsilon u\left(1+|D u|^{2}\right)^{\beta} & \text { in } \Omega, \\ u_{v}=-\cos \theta \sqrt{1+|D u|^{2}} & \text { on } \partial \Omega,\end{cases}
$$

then for $0 \leq \beta<\frac{1}{2}$, using the same calculation we can get the uniform gradient estimates of $u$ for any $\varepsilon>0$. When $\beta=\frac{1}{2}$, we need make a more careful computation in Section 4 so as to get the corresponding existence theorem. 
For a solution $w=w(x)$ to (3.1), it is obvious that $\tilde{w}=w(x)+\lambda t$ solves the following parabolic problem,

$$
\begin{cases}u_{t}=\left(\delta_{i j}-\frac{u_{i} u_{j}}{1+|D u|^{2}}\right) u_{i j} & \text { in } \Omega \times(0, \infty), \\ u_{v}=-\cos \theta \sqrt{1+|D u|^{2}} & \text { on } \partial \Omega \times(0, \infty), \\ u(x, 0)=w(x) & \text { on } \bar{\Omega}\end{cases}
$$

Corollary 3.1. For a solution $u=u(x, t)$ to (1.1), there exists a time-indenpendent positive constant $C$, such that

$$
|u(x, t)-\lambda t| \leq C .
$$

Proof. Let $z(x, t)=u(x, t)-\tilde{w}(x, t)$, then it satisfies the following equation

$$
z_{t}=\tilde{a}_{i j} z_{i j}+b_{i} z_{i}
$$

where $\tilde{a}_{i j}=a_{i j}(D u)$ and $b_{i}=(\tilde{w})_{k l} \int_{0}^{1} a_{k l, p_{i}}(\eta D u+(1-\eta) D \tilde{w}) d \eta$. If $z$ achieves its maximum and minimum on $\bar{\Omega} \times\{0\}$, then

$$
\sup _{\Omega \times(0, \infty)}|u-w-\lambda t|=\sup _{\Omega \times(0, \infty)}|z| \leq \sup _{\Omega \times\{0\}}|z|=\sup _{\Omega}\left|u_{0}-w\right| .
$$

Therefore,

$$
\sup _{\Omega \times(0, \infty)}|u-\lambda t| \leq \sup _{\Omega}|w|+\sup _{\Omega}\left|u_{0}-w\right| .
$$

If $z$ attains its maximum or minimum on $\partial \Omega \times(0, \infty)$, then as in the uniqueness part proof of Theorem 3.1, Hopf's lemma tells us $u_{0}-w$ must be a constant. Therefore $u-\tilde{w}$ also must be a constant on $\Omega \times(0, \infty)$ for the uniqueness of the solution to (1.1), so we have

$$
\sup _{\Omega \times(0, \infty)}|u-\lambda t| \leq \sup _{\Omega \times(0, \infty)}|u-\lambda t-w|+\sup _{\Omega}|w|=\sup _{\Omega}\left|u_{0}-w\right|+\sup _{\Omega}|w| .
$$

Using the technique in [2], the uniform estimates in Lemma 2.1, Theorem 2.1 and Schauder estimates, we get the following result.

Lemma 3.1. Let $u_{1}$ and $u_{2}$ be any two solutions to equation (1.1), with initial data $u_{0,1}$ and $u_{0,2}$ respectively. Let $u=u_{1}-u_{2}$, then $u$ converges to a constant function as $t \rightarrow \infty$. In particular, the limit of any solution to Eq. (1.1) is $\tilde{w}$ up to a constant.

Proof. We now see that $u$ satisfies a linear parabolic equation

$$
\begin{cases}z_{t}=\tilde{a}_{i j} z_{i j}+b_{i} z_{i} & \text { in } \Omega \times(0, \infty), \\ \frac{D_{v} u_{1}}{\sqrt{1+\left|D u_{1}\right|^{2}}}=\frac{D_{v} u_{2}}{\sqrt{1+\left|D u_{2}\right|^{2}}} & \text { on } \partial \Omega \times(0, \infty),\end{cases}
$$


where $\widetilde{a}_{i j}=a_{i j}\left(D u_{1}\right)$ and $b_{i}=\left(u_{2}\right)_{k l} \int_{0}^{1} a_{k l, p_{i}}\left(\eta D u_{1}+(1-\eta) D u_{2}\right) \mathrm{d} \eta$. The strong maximum principle implies that $\operatorname{osc}(u)(t)=\max _{\bar{\Omega}} u(x, t)-\min _{\bar{\Omega}} u(x, t) \geq 0$ is a strictly decreasing function unless $u$ is a constant.

We claim that

$$
\lim _{t \rightarrow \infty} \operatorname{osc}(u)(t)=0 .
$$

Otherwise, if $\lim _{t \rightarrow \infty} O s c(u)(t)=\delta$ for some $\delta>0$, we will reach a contradiction. In fact, given a sequence $t_{n} \rightarrow+\infty$, we define

$$
u_{1, n}(\cdot, t)=u_{1}\left(\cdot, t+t_{n}\right)-\lambda t_{n}
$$

and

$$
u_{2, n}(\cdot, t)=u_{2}\left(\cdot, t+t_{n}\right)-\lambda t_{n}
$$

By Corollary 3, for $i=1,2$, we know $\left|u_{i, n}-\lambda t\right| \leq C$, remark that the uniform(independent of $n$ ) estimates on $\frac{\partial u_{i, n}}{\partial t},\left|D u_{i, n}\right|$ have already been obtained in Lemma 2.1 and Theorem 2.1. According to Schauder theory [21], $u_{1, n}(\cdot, t)$ and $u_{2, n}(\cdot, t)$ are locally (in time) $C^{k}$ uniformly bounded with respect to $n$ for any $k$.

So, there exists a subsequence (still denoted by $t_{n}$ ) such that $u_{1, n}(\cdot, t)$ and $u_{2, n}(\cdot, t)$ converge locally uniformly in any $C^{k}$ to $u_{1}^{*}(\cdot, t)$ and $u_{2}^{*}(\cdot, t)$ respectively. That is

$$
u_{1}^{*}(\cdot, t)=\lim _{n \rightarrow \infty} u_{1, n}(\cdot, t), \quad u_{2}^{*}(\cdot, t)=\lim _{n \rightarrow \infty} u_{2, n}(\cdot, t)
$$

Let $u^{*}=u_{1}^{*}-u_{2}^{*}$, then we deduce that

$$
\begin{aligned}
\operatorname{osc}\left(u^{*}\right)(t) & =\operatorname{osc}\left(u_{1}^{*}-u_{2}^{*}\right) \\
& =\lim _{n \rightarrow \infty} \operatorname{osc}\left(u_{1}\left(x, t+t_{n}\right)-\lambda t_{n}-u_{2}\left(x, t+t_{n}\right)+\lambda t_{n}\right) \\
& =\lim _{n \rightarrow \infty} \operatorname{osc}\left(u_{1}\left(x, t+t_{n}\right)-u_{2}\left(x, t+t_{n}\right)\right) \\
& =\lim _{n \rightarrow \infty} \operatorname{osc}(u)\left(t+t_{n}\right)=\delta,
\end{aligned}
$$

where the second equality holds because of the uniform convergence of $u_{1, n}(\cdot, t)$ and $u_{2, n}(\cdot, t)$.

But $u^{*}$ satisfies the uniformly parabolic equation

$$
\begin{cases}z_{t}=\widetilde{a}_{i j} z_{i j}+b_{i} z_{i} & \text { in } \Omega \times(-\infty, \infty), \\ \frac{D_{v} u_{1}^{*}}{\sqrt{1+\left|D u_{1}^{*}\right|^{2}}}=\frac{D_{v} u_{2}^{*}}{\sqrt{1+\left|D u_{2}^{*}\right|^{2}}} & \text { on } \partial \Omega \times(-\infty, \infty),\end{cases}
$$

where $\widetilde{a}_{i j}=a_{i j}\left(D u_{1}^{*}\right)$ and $b_{i}=\left(u_{2}^{*}\right)_{k l} \int_{0}^{1} a_{k l, p_{i}}\left(\eta D u_{1}^{*}+(1-\eta) D u_{2}^{*}\right) \mathrm{d} \eta$.

By the strong maximum principle and Hopf's lemma, we know $u^{*}$ is a constant. This makes a contradiction to $\csc \left(u^{*}\right)(t) \equiv \delta$ and the claim now is proved. 
According to the claim, we have

$$
\lim _{t \rightarrow \infty} \max _{\bar{\Omega}} u=\lim _{t \rightarrow \infty} \min _{\bar{\Omega}} u=c_{0} \quad \text { for some constant } c_{0} .
$$

It then follows that $\lim _{t \rightarrow \infty}\left|u-c_{0}\right|=0$ and we finish the proof of this lemma.

Proof of Theorem 1.1. From Lemma 2.1 and Theorem 2.1 and Schauder estimate, we obtain uniform estimates in any $C^{k}$-norm for the derivatives of $u$, and locally (in time) uniform bounds for the $C_{0}$ norm. So we get longtime existence with uniform bounds on all higher derivatives of $u$. From Corollary 3 and Lemma 3.1, the limit of any solution to Eq. (1.1) is $\tilde{w}=w+\lambda t$ up to a constant, where $(\lambda, w)$ is the solution to Eq. (3.1) by Theorem 3.1.

\section{Constant mean curvature equation with prescribed contact angle boundary value condition}

In this section, we consider the capillary problem with prescribed contact angle boundary condition. We first obtain the following uniform gradient estimate of $u$ for equations (4.1). Note that, for any fixed $\varepsilon>0$, the existence of solutions to (4.1) is well-known, see [8] for example. Now we can prove the following lemma.

Lemma 4.1. Let $\Omega \subset \mathbb{R}^{n}(n \geq 2)$ be a strictly convex, bounded domain and $\partial \Omega \in C^{3}$. There is a small constant $\varepsilon_{0}$ such that if $\theta \in C^{3}(\bar{\Omega})$ satisfying condition (1.4), and if $u$ is the solution to the following mean curvature type equation with prescribed contact angle boundary condition,

$$
\begin{cases}\operatorname{div}\left(\frac{D u}{\sqrt{1+|D u|^{2}}}\right)=\varepsilon u & \text { in } \Omega, \\ u_{v}=-\cos \theta \sqrt{1+|D u|^{2}} & \text { on } \partial \Omega,\end{cases}
$$

then there exists a constant $C \sim n, \Omega$ such that

$$
\sup _{\bar{\Omega}}|D u| \leq C .
$$

Remark 4.1. The idea and approach for proving Lemma 4.1 are similar with those showed in Theorem 3.1. However, as we remark in Remark 3.2 that there are new difficulties arising here. Moreover, it follows from the remark below Theorem 1 in the work [5] of Concus and Finn that $|u| \leq \frac{C_{1}}{\varepsilon}+C_{2}$. Denoting by $f=\varepsilon u$, we have

$$
\sup _{\bar{\Omega}}|f|=\sup _{\bar{\Omega}}\left|\varepsilon u_{\varepsilon}\right| \leq C_{0}
$$

where $C_{0}$ is independent of $\varepsilon$. One can also derive the same result following the barrier argument in [2] (Just note that there is one more factor $\frac{1}{\sqrt{1+|D u|^{2}}}$ in (4.1) compared to the proof of Theorem 2.6 in [2], which does not affect the barrier argument). 
Proof. Let

$$
\Phi(x)=\log w(x)+\alpha h(x),
$$

where $w(x)=v-\sum_{l=1}^{n} u_{l} h_{l} \cos \theta$ and $\alpha$ is a positive constant to be determined later, $v=$ $\sqrt{1+|D u|^{2}}$. Denoting here by $a_{i j}:=v^{2} \delta_{i j}-u_{i} u_{j}$, Eq. (4.1) now can be expressed to be

$$
\sum_{i, j=1}^{n} a_{i j} u_{i j}=f v^{3}
$$

Assume that $\Phi(x)$ attains the maximum value at $x_{0} \in \bar{\Omega}$. We divide it into the following two cases to complete the proof.

Case 1: If $x_{0} \in \partial \Omega$, this is the same as in Case 1 in Theorem 2.1, since we retain the same boundary condition. By choosing the same $\alpha, \varepsilon_{0}$ as in (2.8), we obtain the same conclusion.

Case 2: If $x_{0} \in \Omega$, we have

$$
\begin{aligned}
& 0=\Phi_{i}=\frac{w_{i}}{w}+\alpha h_{i} \\
& 0 \geq \sum_{i, j=1}^{n} a_{i j} \Phi_{i j}=\sum_{i, j=1}^{n} \frac{a_{i j} w_{i j}}{w}-\sum_{i, j=1}^{n} \alpha^{2} a_{i j} h_{i} h_{j}+\sum_{i, j=1}^{n} \alpha a_{i j} h_{i j}=: \mathrm{I}+\mathrm{II}+\mathrm{III} .
\end{aligned}
$$

We choose a proper coordinates at $\left(x_{0}, t_{0}\right)$ such that $|D u|\left(x_{0}\right)=u_{1}\left(x_{0}\right)>0$ and $\left\{u_{i j}\left(x_{0}\right)\right\}_{2 \leq i, j \leq n}$ is diagonal. Then at $x_{0}, a_{11}=1$ and $a_{i i}=v^{2}$, for $i=2, \cdots, n$.

We always assume that $u_{1}\left(x_{0}\right)$ is large enough in the below computation, such that $u_{1}$, $v$, and $w$ (since we assume $|\cos \theta| \leq \varepsilon_{0}<1$ ) are equivalent to each other at $x_{0}$. Otherwise, we have completed the proof. All the computation below are at the point $x_{0}$.

We start to deal with the terms in (4.5).

$$
\mathrm{II}+\mathrm{III}=\sum_{i, j=1}^{n} \alpha a_{i j} h_{i j}-\sum_{i, j=1}^{n} \alpha^{2} a_{i j} h_{i} h_{j} \geq \alpha k_{1}\left[1+v^{2}(n-1)\right]-\alpha^{2}\left[1+v^{2}\right] .
$$

We denote by $\mathrm{J}:=\sum_{i, j=1}^{n} a_{i j} w_{i j}$. From (4.4), we have

$$
\sum_{l=1}^{n}\left(\frac{u_{l} u_{l i}}{v}-u_{l i} h_{l} \cos \theta-u_{l} h_{l i} \cos \theta+u_{l} h_{l} \sin \theta \theta_{i}\right)=-\alpha h_{i} w
$$

If we denote by

$$
\mathrm{S}_{l}:=\frac{u_{l}}{v}-h_{l} \cos \theta, \quad \text { for } l=1, \cdots, n,
$$

then we obtain

$$
\sum_{l=1}^{n} \mathrm{~S}_{l} u_{l i}=h_{1 i} \cos \theta u_{1}-h_{1} \sin \theta \theta_{i} u_{1}-\alpha h_{i} w
$$


We also have

$$
\begin{aligned}
& u_{1 i}=-\frac{\mathrm{S}_{i}}{\mathrm{~S}_{1}} u_{i i}+\frac{1}{\mathrm{~S}_{1}} \sum_{l=1}^{n} \mathrm{~S}_{l} u_{l i}, \quad \text { for } i=2, \cdots, n, \\
& u_{11}=-\sum_{k=2}^{n} \frac{\mathrm{S}_{k}}{\mathrm{~S}_{1}} u_{1 k}+\frac{1}{\mathrm{~S}_{1}} \sum_{l=1}^{n} \mathrm{~S}_{l} u_{l 1}=\sum_{i=2}^{n} \frac{\mathrm{S}_{i}^{2}}{\mathrm{~S}_{1}^{2}} u_{i i}-\sum_{i=2}^{n} \frac{\mathrm{S}_{i}}{\mathrm{~S}_{1}^{2}}\left(\sum_{l=1}^{n} \mathrm{~S}_{l} u_{l i}\right)+\frac{1}{\mathrm{~S}_{1}} \sum_{l=1}^{n} \mathrm{~S}_{l} u_{l 1} .
\end{aligned}
$$

From the equation we have,

$$
f v=\frac{u_{11}}{v^{2}}+\sum_{i=2}^{n} u_{i i}
$$

On the other hand, since

$$
f v^{3}=\sum_{i, j=1}^{n}\left(v^{2} \delta_{i j}-u_{i} u_{j}\right) u_{i j}=v^{2} \Delta u-u_{1}^{2} u_{11}
$$

we have

$$
3 f v-2 \Delta u=f v-2 \frac{u_{1}^{2}}{v^{2}} u_{11}=\left(\frac{1}{v^{2}}-2 \frac{u_{1}^{2}}{v^{2}}\right) u_{11}+\sum_{i=2}^{n} u_{i i}
$$

Substituting (4.9) and (4.10) into (4.11), we obtain

$$
\begin{aligned}
& 3 f v-2 \Delta u \\
= & \sum_{i=2}^{n}\left(\frac{1}{v^{2}}-\frac{2 u_{1}^{2}}{v^{2}}\right) \frac{S_{i}^{2}}{\mathrm{~S}_{1}^{2}} u_{i i}+\sum_{i=2}^{n} u_{i i}-\sum_{i=2}^{n}\left(\frac{1}{v^{2}}-\frac{2 u_{1}^{2}}{v^{2}}\right) \frac{\mathrm{S}_{i}}{\mathrm{~S}_{1}^{2}}\left(\sum_{l=1}^{n} \mathrm{~S}_{l} u_{l i}\right)+\left(\frac{1}{v^{2}}-\frac{2 u_{1}^{2}}{v^{2}}\right) \frac{1}{\mathrm{~S}_{1}} \sum_{l=1}^{n} \mathrm{~S}_{l} u_{l 1} .
\end{aligned}
$$

As in Section 2, by (2.18) and $\mathrm{J}:=\sum_{i, j=1}^{n} a_{i j} w_{i j}$ we have

$$
\begin{aligned}
& \mathrm{J}= \sum_{i, j, k=1}^{n} a_{i j}\left(\frac{u_{k i} u_{k j}}{v}-\sum_{l=1}^{n} \frac{u_{k} u_{k i} u_{l} u_{l j}}{v^{3}}\right)+\sum_{i, j, k=1}^{n} a_{i j}\left(\frac{u_{k} u_{k i j}}{v}-u_{k i j} h_{k} \cos \theta\right) \\
&-\sum_{i, j, k=1}^{n} a_{i j} u_{k}\left(h_{k i j} \cos \theta-2 h_{k i} \sin \theta \theta_{j}-h_{k} \cos \theta \theta_{i} \theta_{j}-h_{k} \sin \theta \theta_{i j}\right) \\
& \quad-2 \sum_{i, j, k=1}^{n} a_{i j} u_{k i}\left(h_{k j} \cos \theta-h_{k} \sin \theta \theta_{j}\right) \\
&=: \mathrm{J}_{1}+\mathrm{J}_{2}+\mathrm{J}_{3}+\mathrm{J}_{4},
\end{aligned}
$$


where $\mathrm{J}_{1}, \mathrm{~J}_{3}$ and $\mathrm{J}_{4}$ are almost the same as in the proof of Theorem 2.1. We have

$$
\begin{aligned}
\mathrm{J}_{1} & =\sum_{i, j, k=1}^{n} a_{i j}\left(\frac{u_{k i} u_{k j}}{v}-\sum_{l=1}^{n} \frac{u_{k} u_{k i} u_{l} u_{l j}}{v^{3}}\right)=\frac{1}{v^{3}} u_{11}^{2}+\frac{2}{v} \sum_{i=2}^{n} u_{1 i}^{2}+v \sum_{i=2}^{n} u_{i i}^{2} \\
\mathrm{~J}_{3} & =-\sum_{i, j, k=1}^{n} a_{i j} u_{k}\left(h_{k i j} \cos \theta-2 h_{k i} \sin \theta \theta_{j}-h_{k} \cos \theta \theta_{i} \theta_{j}-h_{k} \sin \theta \theta_{i j}\right) \\
& \geq-C\left(|\cos \theta|+|D \theta|+\left|D^{2} \theta\right|\right) v^{3}, \\
\mathrm{~J}_{4} & =-2 \sum_{i, j, k=1}^{n} a_{i j} u_{k i}\left(h_{k j} \cos \theta-h_{k} \sin \theta \theta_{j}\right) \\
& =(|\cos \theta|+|D \theta|)\left[O\left(v^{2}\right)+O(v)\right] \sum_{i=2}^{n}\left|u_{i i}\right|+O\left(v^{2} u_{1}\right)(|\cos \theta|+|D \theta|) \\
& \geq-\frac{v}{4} \sum_{i=2}^{n} u_{i i}^{2}-C(|\cos \theta|+|D \theta|) v^{3} .
\end{aligned}
$$

A direct computation gives

$$
\begin{aligned}
\mathrm{J}_{2} & :=\sum_{i, j, k=1}^{n} a_{i j}\left(\frac{u_{k} u_{k i j}}{v}-u_{k i j} h_{k} \cos \theta\right)=\sum_{i, j, l=1}^{n} a_{i j} \mathrm{~S}_{l} u_{l i j} \\
& =\sum_{i, j, l=1}^{n} \mathrm{~S}_{l}\left(a_{i j} u_{i j}\right)_{l}-\sum_{i, j, l=1}^{n} \mathrm{~S}_{l} a_{i j, l} u_{i j}=\sum_{l=1}^{n} \mathrm{~S}_{l}\left(f v^{3}\right)_{l}-\sum_{i, j, l=1}^{n} \mathrm{~S}_{l} a_{i j, l} u_{i j} \\
& =\sum_{l=1}^{n} \mathrm{~S}_{l} f_{l} v^{3}+3 \sum_{k, l=1}^{n} \mathrm{~S}_{l} f v u_{k} u_{k l}-2 \sum_{k, l=1}^{n} \mathrm{~S}_{l} u_{k} u_{k l} \Delta u+\sum_{i, j, l=1}^{n} 2 \mathrm{~S}_{l} u_{i l} u_{j} u_{i j} \\
& =u_{1}(3 f v-2 \Delta u) \sum_{l=1}^{n} \mathrm{~S}_{l} u_{1 l}+2 u_{11} u_{1} \sum_{l=1}^{n} \mathrm{~S}_{l} u_{1 l}+2 \sum_{i=2}^{n} u_{1 i} u_{1}\left(\sum_{l=1}^{n} \mathrm{~S}_{l} u_{i l}\right)+\sum_{l=1}^{n} \mathrm{~S}_{l} f_{l} v^{3} \\
& =\mathrm{J}_{21}+\mathrm{J}_{22}+\mathrm{J}_{23}+\mathrm{J}_{24} .
\end{aligned}
$$

Note that $f_{l}=\varepsilon u_{l}$, and $S_{1}=\frac{u_{1}}{v}-h_{1} \cos \theta$, suppose that $|\cos \theta|<\frac{1}{2}$, we have

$$
\mathrm{J}_{24}=\sum_{l=1}^{n} \mathrm{~S}_{l} f_{l} v^{3}=\mathrm{S}_{1} \varepsilon u_{1} v^{3}>0
$$

It follows from (4.12) that

$$
\begin{aligned}
\mathrm{J}_{21}= & u_{1} \sum_{l=1}^{n} \mathrm{~S}_{l} u_{1 l}(3 f v-2 \Delta u) \\
= & u_{1}\left(\sum_{l=1}^{n} \mathrm{~S}_{l} u_{1 l}\right)\left[\sum_{i=2}^{n}\left(\frac{1}{v^{2}}-\frac{2 u_{1}^{2}}{v^{2}}\right) \frac{\mathrm{S}_{i}^{2}}{\mathrm{~S}_{1}^{2}} u_{i i}+\sum_{i=2}^{n} u_{i i}\right] \\
& \quad-u_{1}\left(\sum_{m=1}^{n} \mathrm{~S}_{m} u_{1 m}\right)\left[\sum_{i=2}^{n}\left(\frac{1}{v^{2}}-\frac{2 u_{1}^{2}}{v^{2}}\right) \frac{\mathrm{S}_{i}}{\mathrm{~S}_{1}^{2}}\left(\sum_{l=1}^{n} \mathrm{~S}_{l} u_{l i}\right)-\left(\frac{1}{v^{2}}-\frac{2 u_{1}^{2}}{v^{2}}\right) \frac{1}{\mathrm{~S}_{1}}\left(\sum_{l=1}^{n} \mathrm{~S}_{l} u_{l 1}\right)\right] .
\end{aligned}
$$


Using (4.9), we have

$$
\begin{aligned}
\mathrm{J}_{22} & =2 \sum_{l=1}^{n} \mathrm{~S}_{l} u_{1 l} u_{1} u_{11} \\
& =2\left(\sum_{l=1}^{n} \mathrm{~S}_{l} u_{1 l}\right) u_{1}\left(\sum_{i=2}^{n} \frac{\mathrm{S}_{i}^{2}}{\mathrm{~S}_{1}^{2}} u_{i i}\right)-2\left(\sum_{m=1}^{n} \mathrm{~S}_{m} u_{1 m}\right) u_{1}\left[\sum_{i=2}^{n} \frac{\mathrm{S}_{i}}{\mathrm{~S}_{1}^{2}}\left(\sum_{l=1}^{n} \mathrm{~S}_{l} u_{l i}\right)-\frac{1}{\mathrm{~S}_{1}}\left(\sum_{l=1}^{n} \mathrm{~S}_{l} u_{l 1}\right)\right] .
\end{aligned}
$$

Using (4.8), we have

$$
\mathrm{J}_{23}=2 \sum_{i=2}^{n} u_{1 i} u_{1}\left(\sum_{l=1}^{n} \mathrm{~S}_{l} u_{i l}\right)=-2 \sum_{i=2}^{n} u_{1} \frac{\mathrm{S}_{i}}{\mathrm{~S}_{1}} u_{i i}\left(\sum_{l=1}^{n} \mathrm{~S}_{l} u_{i l}\right)+2 \sum_{i=2}^{n} \frac{u_{1}}{\mathrm{~S}_{1}}\left(\sum_{l=1}^{n} \mathrm{~S}_{l} u_{i l}\right)^{2} .
$$

Substituting (4.18)-(4.21) into (4.17), we obtain

$$
\begin{aligned}
\mathrm{J}_{2} \geq u_{1} & \left(\sum_{l=1}^{n} \mathrm{~S}_{l} u_{1 l}\right)\left[\sum_{i=2}^{n}\left(\frac{1}{v^{2}}-\frac{2 u_{1}^{2}}{v^{2}}\right) \frac{\mathrm{S}_{i}^{2}}{\mathrm{~S}_{1}^{2}} u_{i i}+\sum_{i=2}^{n} u_{i i}\right] \\
& -u_{1}\left(\sum_{m=1}^{n} \mathrm{~S}_{m} u_{1 m}\right)\left[\sum_{i=2}^{n}\left(\frac{1}{v^{2}}-\frac{2 u_{1}^{2}}{v^{2}}\right) \frac{\mathrm{S}_{i}}{\mathrm{~S}_{1}^{2}}\left(\sum_{l=1}^{n} \mathrm{~S}_{l} u_{l i}\right)-\left(\frac{1}{v^{2}}-\frac{2 u_{1}^{2}}{v^{2}}\right) \frac{1}{\mathrm{~S}_{1}}\left(\sum_{l=1}^{n} \mathrm{~S}_{l} u_{l 1}\right)\right] \\
& +2\left(\sum_{l=1}^{n} \mathrm{~S}_{l} u_{1 l}\right) u_{1}\left(\sum_{i=2}^{n} \frac{\mathrm{S}_{i}^{2}}{\mathrm{~S}_{1}^{2}} u_{i i}\right)-2\left(\sum_{m=1}^{n} \mathrm{~S}_{m} u_{1 m}\right) u_{1}\left[\sum_{i=2}^{n} \frac{\mathrm{S}_{i}}{\mathrm{~S}_{1}^{2}}\left(\sum_{l=1}^{n} \mathrm{~S}_{l} u_{l i}\right)-\frac{1}{\mathrm{~S}_{1}}\left(\sum_{l=1}^{n} \mathrm{~S}_{l} u_{l 1}\right)\right] \\
& -2 \sum_{i=2}^{n} u_{1} \frac{\mathrm{S}_{i}}{\mathrm{~S}_{1}} u_{i i}\left(\sum_{l=1}^{n} \mathrm{~S}_{l} u_{i l}\right)+2 \sum_{i=2}^{n} \frac{u_{1}}{\mathrm{~S}_{1}}\left(\sum_{l=1}^{n} \mathrm{~S}_{l} u_{i l}\right)^{2} \\
= & \frac{3 u_{1}}{v^{2}}\left(\sum_{l=1}^{n} \mathrm{~S}_{l} u_{1 l}\right)\left(\sum_{i=2}^{n} \frac{\mathrm{S}_{i}^{2}}{\mathrm{~S}_{1}^{2}} u_{i i}\right)+\frac{3 u_{1}}{v^{2}} \frac{1}{\mathrm{~S}_{1}}\left(\sum_{l=1}^{n} \mathrm{~S}_{l} u_{l 1}\right)^{2}-\frac{3 u_{1}}{v^{2}}\left(\sum_{m=1}^{n} \mathrm{~S}_{m} u_{1 m}\right) \sum_{i=2}^{n} \frac{\mathrm{S}_{i}}{\mathrm{~S}_{1}^{2}}\left(\sum_{l=1}^{n} \mathrm{~S}_{l} u_{l i}\right) \\
& +u_{1}\left(\sum_{l=1}^{n} \mathrm{~S}_{l} u_{1 l}\right)\left(\sum_{i=2}^{n} u_{i i}\right)-2 u_{1} \sum_{i=2}^{n} \frac{\mathrm{S}_{i}}{\mathrm{~S}_{1}} u_{i i}\left(\sum_{l=1}^{n} \mathrm{~S}_{l} u_{i l}\right)+2 \sum_{i=2}^{n} \frac{u_{1}}{\mathrm{~S}_{1}}\left(\sum_{l=1}^{n} \mathrm{~S}_{l} u_{i l}\right)^{2} \\
= & \mathrm{K}_{1}+\mathrm{K}_{2}+\mathrm{K}_{3}+\mathrm{K}_{4}+\mathrm{K}_{5}+\mathrm{K}_{6} .
\end{aligned}
$$

Using the fact $a x^{2}+b x \geq-\frac{b^{2}}{4 a}$ for any $a>0$, we obtain

$$
\begin{aligned}
& \mathrm{K}_{1}+\mathrm{K}_{2}+\mathrm{K}_{5}+\mathrm{K}_{6} \\
= & \frac{3 u_{1}}{v^{2}}\left(\sum_{l=1}^{n} \mathrm{~S}_{l} u_{1 l}\right)\left(\sum_{i=2}^{n} \frac{\mathrm{S}_{i}^{2}}{\mathrm{~S}_{1}^{2}} u_{i i}\right)+\frac{3 u_{1}}{v^{2}}\left(\frac{1}{\mathrm{~S}_{1}} \sum_{l=1}^{n} \mathrm{~S}_{l} u_{l 1}\right)^{2} \\
& \quad-2 u_{1} \sum_{i=2}^{n} \frac{\mathrm{S}_{i}}{\mathrm{~S}_{1}} u_{i i}\left(\sum_{l=1}^{n} \mathrm{~S}_{l} u_{i l}\right)+2 \sum_{i=2}^{n} \frac{u_{1}}{\mathrm{~S}_{1}}\left(\sum_{l=1}^{n} \mathrm{~S}_{l} u_{i l}\right)^{2} \\
\geq & -\frac{3(n-1) u_{1}}{4 v^{2}} \sum_{i=2}^{n} \frac{\mathrm{S}_{i}^{4}}{\mathrm{~S}_{1}^{2}} u_{i i}^{2}-\frac{u_{1}}{2} \sum_{i=2}^{n} \frac{\mathrm{S}_{i}^{2}}{\mathrm{~S}_{1}} u_{i i}^{2} .
\end{aligned}
$$


Noting that $S_{i}=O(\cos \theta)$ for $i \geq 2, u_{1}, v$ are comparable provided $u_{1} \geq 1$, and by (4.7) we see that.

$$
\left.\mathrm{K}_{3}=-\frac{3 u_{1}}{v^{2}}\left(\sum_{l=1}^{n} \mathrm{~S}_{l} u_{1 l}\right) \sum_{i=2}^{n} \frac{\mathrm{S}_{i}}{\mathrm{~S}_{1}^{2}}\left(\sum_{l=1}^{n} \mathrm{~S}_{l} u_{l i}\right) \geq-C(|\cos \theta|+|D \theta|)+|\alpha|\right) v
$$

Now we deal with term $\mathrm{K}_{4}$, by (4.7), we have

$$
\begin{aligned}
\left|\mathrm{K}_{4}\right| & =\left|u_{1}\left(\sum_{l=1}^{n} \mathrm{~S}_{l} u_{1 l}\right)\left(\sum_{i=2}^{n} u_{i i}\right)\right| \\
& \leq\left|\left(h_{11} \cos \theta-h_{1} \sin \theta \theta_{1}\right)\right| u_{1}^{2} \sum_{i=2}^{n}\left|u_{i i}\right|+\left|\alpha h_{1} u_{1} w \sum_{i=2}^{n} u_{i i}\right| \\
& \leq C(|\cos \theta|+|D \theta|) v^{3}+C(|\cos \theta|+|D \theta|) v \sum_{i=2}^{n} u_{i i}^{2}+\frac{u_{1}}{4} \sum_{i=2}^{n} u_{i i}^{2}+(n-1) \alpha^{2} u_{1} w^{2} .
\end{aligned}
$$

Thus we have

$$
\begin{gathered}
\mathrm{J}_{2} \geq-\frac{3(n-1) u_{1}}{4 v^{2}} \sum_{i=2}^{n} \frac{S_{i}^{4}}{\mathrm{~S}_{1}^{2}} u_{i i}^{2}-\frac{u_{1}}{2} \sum_{i=2}^{n} \frac{\mathrm{S}_{i}^{2}}{\mathrm{~S}_{1}} u_{i i}^{2}-\frac{u_{1}}{3} \sum_{i=2}^{n} u_{i i}^{2}-\alpha^{2}(n-1) u_{1} w^{2} \\
-C(|\cos \theta|+|D \theta|) v^{3}-C(|\cos \theta|+|D \theta|+|\alpha|) v .
\end{gathered}
$$

Substituting (4.14), (4.23), (4.15) and (4.16) into (4.13), we have

$$
\begin{aligned}
& \mathrm{J} \geq \frac{1}{v^{3}} u_{11}^{2}+\frac{2}{v} \sum_{i=2}^{n} u_{1 i}^{2}+v \sum_{i=2}^{n} u_{i i}^{2}-\frac{v}{4} \sum_{i=2}^{n} u_{i i}^{2} \\
&-\frac{3(n-1) u_{1}}{4 v^{2}} \sum_{i=2}^{n} \frac{\mathrm{S}_{i}^{4}}{\mathrm{~S}_{1}^{2}} u_{i i}^{2}-\frac{u_{1}}{2} \sum_{i=2}^{n} \frac{\mathrm{S}_{i}^{2}}{\mathrm{~S}_{1}} u_{i i}^{2}-\frac{u_{1}}{3} \sum_{i=2}^{n} u_{i i}^{2}-\alpha^{2}(n-1) u_{1} w^{2} \\
&-C\left(|\cos \theta|+|D \theta|+\left|D^{2} \theta\right|\right) v^{3}-C(|\cos \theta|+|D \theta|+|\alpha|) v \\
& \geq-C\left(|\cos \theta|+|D \theta|+\left|D^{2} \theta\right|\right) v^{3}-\alpha^{2}(n-1) u_{1} w^{2},
\end{aligned}
$$

where we take $|\cos \theta| \leq \frac{1}{100}$ such that $\frac{1}{2} \frac{S_{i}^{2}}{S_{1}} \leq \frac{1}{4}$.

Note that $\frac{v}{2}<w<\frac{3 v}{2}$, we have

$$
\mathrm{I}:=\frac{\mathrm{J}}{w} \geq-2 C\left(|\cos \theta|+|D \theta|+\left|D^{2} \theta\right|\right) v^{2}-2 n \alpha^{2} v^{2} .
$$

Substituting (4.25) and (4.6) into (4.5), we obtain

$$
0 \geq \mathrm{I}+\mathrm{II}+\mathrm{III} \geq \alpha k_{1}\left[1+v^{2}(n-1)\right]-\alpha^{2}\left[1+v^{2}\right]-2 C\left(|\cos \theta|+|D \theta|+\left|D^{2} \theta\right|\right) v^{2}-2 n \alpha^{2} v^{2} .
$$


By taking $\alpha=\min \left\{\frac{k_{1}}{8}, \frac{\kappa_{0}}{3}, 1\right\}:=\alpha_{0}$ and $\varepsilon_{0}:=\min \left\{\frac{\kappa_{0}}{9\left(M_{1}+3\right)}, \frac{\alpha_{0} k_{1}}{16 C_{1}}, \frac{1}{100}\right\}$ in (1.4), we obtain

$$
v\left(x_{0}\right) \leq \tilde{C} .
$$

Finally, combining all above two cases together, thus we have $v\left(x_{0}\right) \leq \tilde{C}$, where $\tilde{C}$ is independent of $\varepsilon$ and $\|u\|_{C^{0}}$.

With the preparations above, now we are ready to prove Theorem 1.3.

Proof of Theorem 1.3. To solve Eq. (1.7), we will estimate the solutions to the following family of equations $\left(\star_{\varepsilon, L}\right)$.

$$
\begin{cases}\operatorname{div}\left(\frac{D u}{\sqrt{1+|D u|^{2}}}\right)=\varepsilon u-L & \text { in } \Omega, \\ u_{v}=-\cos \theta \sqrt{1+|D u|^{2}} & \text { on } \partial \Omega,\end{cases}
$$

for any given $\varepsilon \in(0,1)$ and $L \in \mathbb{R}$.

Firstly, from [19] or [20], we know that there exists a unique solution $u_{\varepsilon, 0}$ to problem $\left(\star_{\varepsilon, 0}\right)$ for $L=0$ and any $\varepsilon>0$. Hence for general $L \in \mathbb{R}$, one denotes $u_{\varepsilon, L}(x):=u_{\varepsilon, 0}(x)+\frac{L}{\varepsilon}$, thus it solves $\left(\star_{\varepsilon, L}\right)$ and also be the unique solution of problem $\left(\star_{\varepsilon, L}\right)$.

Secondly, we show that, for any $\varepsilon \in(0,1)$, there exists a unique constant $L_{\varepsilon}$ satisfying $\left\|u_{\varepsilon, L_{\varepsilon}}\right\|_{C^{1}(\bar{\Omega})} \leq C$, where $C$ is independent of $\varepsilon$. In fact, one can achieve this by just constructing the supersolution and subsolution of $\left(\star_{\varepsilon, 0}\right)$. Let $\psi$ be the smooth fixed function satisfying

$$
\psi_{v}=-\cos \theta \sqrt{1+|D \psi|^{2}} \text { on } \partial \Omega \text { and } \psi \in C^{\infty}(\bar{\Omega}) .
$$

Denotes $M:=\sup _{\bar{\Omega}}|\psi|+\sup _{\bar{\Omega}}\left|\operatorname{div}\left(\frac{D \psi}{\sqrt{1+|D \psi|^{2}}}\right)\right|+1$, we consider the functions $\psi_{ \pm}(x):=$ $\psi(x) \pm \frac{M}{\varepsilon}$. It follows that, for any $\varepsilon \in(0,1)$, we have

$$
\begin{aligned}
\mathcal{L}\left(u_{\varepsilon, 0}-\psi_{+}\right) & :=\operatorname{div}\left(\frac{D u_{\varepsilon, 0}}{\sqrt{1+\left|D u_{\varepsilon, 0}\right|^{2}}}\right)-\operatorname{div}\left(\frac{D \psi}{\sqrt{1+|D \psi|^{2}}}\right) \\
& \geq \varepsilon u_{\varepsilon, 0}-\varepsilon \psi-M=\varepsilon\left(u_{\varepsilon, 0}-\psi_{+}\right),
\end{aligned}
$$

where the elliptic operator $\mathcal{L} u:=\sum_{i, j=1}^{n} \frac{\partial}{\partial x_{i}}\left(\hat{a}_{j}^{i}(x) \partial_{x_{j}} u\right)$ with

$$
\hat{a}_{j}^{i}(x):=\int_{0}^{1} \partial_{p_{j}} A^{i}\left(t D u_{\varepsilon, 0}(x)+(1-t) D \psi(x)\right) \mathrm{d} t, \quad A^{i}(p):=\frac{p_{i}}{\sqrt{1+|p|^{2}}} \text { for } 1 \leq i \leq n .
$$

Therefore, the maximum principle implies that $u_{\varepsilon, 0}-\psi_{+}$attains the nonnegative maximum value at the boundary, say $x_{0} \in \partial \Omega$ and $u_{\varepsilon, 0}\left(x_{0}\right)-\psi_{+}\left(x_{0}\right) \geq 0$. Since

$$
\frac{D_{v} \psi}{\sqrt{1+|D \psi|^{2}}}=\frac{D_{\nu} u_{\varepsilon, 0}}{\sqrt{1+\left|D u_{\varepsilon, 0}\right|^{2}}}
$$


by using the similar argument in Altschuler-Wu (see Theorem 2.6 in [2]) or the proof of Theorem 3.1, it yields a contradiction. Hence $u_{\varepsilon, 0} \leq \psi_{+}$in $\bar{\Omega}$. Similarly, from

$$
\begin{aligned}
\mathcal{L}\left(u_{\varepsilon, 0}-\psi_{-}\right) & :=\operatorname{div}\left(\frac{D u_{\varepsilon, 0}}{\sqrt{1+\left|D u_{\varepsilon, 0}\right|^{2}}}\right)-\operatorname{div}\left(\frac{D \psi}{\sqrt{1+|D \psi|^{2}}}\right) \\
& \leq \varepsilon u_{\varepsilon, 0}-\varepsilon \psi+M=\varepsilon\left(u_{\varepsilon, 0}-\psi_{-}\right),
\end{aligned}
$$

using the maximum principle and the boundary condition again, it follows that $u_{\varepsilon, 0} \geq \psi_{-}$ in $\bar{\Omega}$. Consequently, we have

$$
u_{\varepsilon,-M}(x)=u_{\varepsilon, 0}(x)-\frac{M}{\varepsilon} \leq \psi(x) \leq u_{\varepsilon, 0}(x)+\frac{M}{\varepsilon}=u_{\varepsilon, M}(x) \quad \text { in } \quad \bar{\Omega} .
$$

Since $u_{\varepsilon, L}$ is strictly increasing with respect to $L \in \mathbb{R}$ for any fixed $\varepsilon \in(0,1)$, it follows that, there exists a unique constant $L_{\varepsilon} \in[-M, M]$ such that $u_{\varepsilon, L_{\varepsilon}}\left(x_{0}\right)=\psi\left(x_{0}\right)$ for some $x_{0} \in \Omega$. Combining this with Lemma 4.1, it yields that we also have the uniform $C^{0}$ estimate for $u_{\varepsilon, L_{\varepsilon}}$, then we obtain $\left\|u_{\varepsilon, L_{\varepsilon}}\right\|_{C^{1}(\bar{\Omega})} \leq C$, where $C$ is independent of $\varepsilon$. Following the standard estimates [11] (Theorem 13.2), it implies that $\left\|u_{\varepsilon, L_{\varepsilon}}\right\|_{C^{k, \alpha}(\bar{\Omega})} \leq C$ for any $k \in \mathbb{N}$, where $\alpha=\alpha(n, \bar{\Omega})$. Hence, by the Arzela-Ascoli theorem and (4.3), there exists $\varepsilon_{i} \rightarrow 0$, a subsequence $u_{\varepsilon_{i}, L_{\varepsilon_{i}}}, L_{\varepsilon_{i}}$, and a smooth function $u_{\infty}$ such that

$$
\varepsilon_{i} u_{\varepsilon_{i}, L_{\varepsilon_{i}}}-L_{\varepsilon_{i}} \rightarrow \tau, \text { and } u_{\varepsilon_{i}, L_{\varepsilon_{i}}} \rightarrow u_{\infty}
$$

And one can easily see that $\left(u_{\infty}, \tau\right)$ solves (1.7).

As in the proof of Theorem 3.1 (or see [2], Theorem 2.6 there) we can get the uniqueness. Thus we have completed the proof.

\section{Acknowledgments}

The second and the third author are supported by the National Natural Science Foundation of China (Grant No. 11471188). The second author is also supported by the National Natural Science Foundation of China (Grant No. 11721101) and the National Natural Science Foundation of China (Grant No. 11871255).

\section{References}

[1] S. J. Altschuler and L. F. Wu, Convergence to translating solutions for a class of quasilinear parabolic boundary problems, Math. Ann., 295(4) (1993), 761-765.

[2] S. J. Altschuler and L. F. Wu, Translating surfaces of the non-parametric mean curvature flow with prescribed contact angle, Calc. Var. Partial Differential Equations, 2(1) (1994), 101-111.

[3] G. Bellettini and S. Y. Kholmatov, Minimizing movements for mean curvature flow of droplets with prescribed contact angle, J. Math. Pures Appl., 117(9) (2018), 1-58. 
[4] L. Caffarelli, L. Nirenberg and J. Spruck, Dirichlet problem for nonlinear second order elliptic equations III, Functions of the eigenvalues of the Hessian, Acta Math., 155 (1985), 261-301.

[5] P. Concus and R. Finn, On capillary free surfaces in a gravitational field, Acta Math., 132 (1974), 207-223.

[6] P. Concus and R. Finn, On capillary free surfaces in the absence of gravity, Acta Math., 132(1) (1974), 177-198.

[7] R. Finn, Existence and nonexistence of capillary surfaces, Manuscripta Math., 28(1-3) (1979), $1-11$.

[8] R. Finn, Equilibrium Capillary Surfaces, Springer-Verlag, New York, 1986.

[9] R. Finn and E. Giusti, Nonexistence and existence of capillary surfaces, Manuscripta Math., 28(1-3) (1979), 13-20.

[10] C. Gerhardt, Global regularity of the solutions to the capillarity problem, Ann. Scuola Norm. Sup. Pisa Cl Sci., 3(1) (1976), 157-175.

[11] D. Gilbarg and N. S. Trudinger, Elliptic Partial Differential Equations of Second Order, Reprint of the 1998 edition, Classics in Mathematics, Springer-Verlag, Berlin, 2001.

[12] E. Giusti, Boundary value problems for non-parametric surfaces of prescribed mean curvature, Ann. Scuola Norm. Sup. Pisa Cl Sci., 3(3) (1976), 501-548.

[13] E. Giusti, On the equation of surfaces of prescribed mean curvature, Existence and uniqueness without boundary conditions, Invent Math., 46(2) (1978), 111-137.

[14] B. Guan, Mean curvature motion of non-parametric hypersurfaces with contact angle condition, Elliptic and Parabolic Methods in Geometry, AK Peters, Wellesley (MA), (1996), 47-56.

[15] G. Huisken, Nonparametric mean curvature evolution with boundary conditions, J. Differential Equations, 77(2) (1989), 369-378.

[16] G. T. Huisken and T. Ilmanen, The inverse mean curvature flow and the Riemannian Penrose inequality, J. Differential Geom., 59(3) (2001), 353-437.

[17] H. Ishii, A short introduction to viscosity solutions and the large time behavior of solutions of Hamilton-Jacobi equations, Hamilton-Jacobi Equations: Approximations, Numerical Analysis and Applications, 111-249, Lecture Notes in Math, 2014, Springer, Heidelberg, 2013.

[18] M. Katsoulakis, G. T. Kossioris and F. Reitich, Generalized motion by mean curvature with Neumann conditions and the Allen-Cahn model for phase transitions, J. Geom. Anal., 5(2) (1995), 255-279.

[19] N. J. Korevaar, Maximum principle gradient estimates for the capillary problem, Commun. Partial Differential Equations, 13(1) (1988), 1-31.

[20] G. M. Lieberman, Gradient estimates for capillary-type problems via the maximum principle, Commun. Partial Differential Equations, 13(1) (1988), 33-59.

[21] G. M. Lieberman, Second Order Parabolic Differential Equations, World scientific, 1996.

[22] G. M. Lieberman, Oblique Boundary Value Problems for Elliptic Equations, World Scientific Publishing, 2013.

[23] G. M. Lieberman, Zero gravity capillary surfaces and integral estimates, variational analysis and applications, Nonconvex Optim. Appl., 79 (2005), 633-649.

[24] X. N. Ma and P. H. Wang, Boundary gradient estimate of the solution to mean curvature equations with Neumann boundary or prescribed contact angle boundary (in Chinese), Sci. Sin. Math., 48 (2018), 213-226, doi:10.1360/N012017-00071.

[25] X. N. Ma, P. H. Wang and W. Wei, Constant mean curvature surfaces and mean curvature flow with non-zero Neumann boundary conditions on strictly convex domains, J. Funct. 
Anal., 274(1) (2018), 252-277.

[26] V. I. Oliker and N. N. Uraltseva, Evolution of nonparametric surfaces with speed depending on curvature II, The mean curvature case, Commun. Pure Appl. Math., 46(1) (1993), 97-135.

[27] G. H. Qiu and C. Xia, Classical Neumann problems for Hessian equations and AlexandrovFenchel's inequalities, Int. Math. Res. Not., 20 (2019), 6285-6303.

[28] L. Simonand J. Spruck, Existence and regularity of a capillary surface with prescribed contact angle, Arch. Rational Mech. Anal., 61(1) (1976), 19-34.

[29] N. Ural'tseva, Solvability of the capillary problem, Vestnik Leningrad Univ, 19 (1973), 54-64; 1 (1975), 143-149. English Translation in vestnik Leningrad Univ Math., 6 (1979), 363-375; 8 (1980), 151-158.

[30] H. Zhou, Nonparametric mean curvature type flows of graphs with contact angle conditions, Int. Math. Res. Not., 19 (2018), 6026-6069. 